\title{
PROPERTIES OF THE GRAPH TOPOLOGY FOR SCALAR TRANSFER FUNCTIONS*
}

\author{
THOMAS S. BRINSMEAD ${ }^{\dagger}$ AND BRIAN D. O. ANDERSON ${ }^{\dagger}$
}

\begin{abstract}
Necessary and sufficient conditions for the existence of a scalar homotopy in the Vinnicombe metric between two given transfer functions are presented. Previous results show that for multivariable transfer functions, the existence of a homotopy is related to a winding number condition on the homotopy end-points. Here, it is shown that for scalar transfer functions the existence of a homotopy is also related to a Cauchy index condition. Extensive use is made of the relationship, previously noted by Brockett, between the Cauchy index of a real function and the argument of a particular related complex function.
\end{abstract}

Keywords. Vinnicombe Metric, Nu-gap Metric, Scalar Homotopy, Subunitary Homotopy, Cauchy Index

1. Introduction. A desire to analyse robustness of controlled performance has motivated the development of various control-relevant metrics on linear operator spaces corresponding to transfer function matrices. For linear systems, it is known that the graph metric (also known as the gap metric [8]) and the $\nu$-gap (Nu-gap or Vinnicombe gap) metric $[14,17]$ induce the weakest operator topologies under which both stability and performance are robust properties $[6]^{1}$. In addition, various extensions of such metrics to nonlinear operator spaces have also been proposed $[2,3,11,12,15]$.

The definition of the Nu-gap metric for linear operators involves checking a property known as the "winding number condition" $[14,17]$ related to counting encirclements of the origin of a particular frequency domain function. Because it is defined in terms of an operator in the frequency domain, such a condition is an inherently linear systems concept. If the Vinnicombe metric is to be extended to nonlinear systems, then an obvious stepping stone is to characterise the winding number condition in a way that does not depend upon the linearity of the underlying operators.

That the winding number condition is equivalent to the existence of a homotopy between two systems has been known for some time [17] (see Section 7.2). However, as pointed out by a reviewer, the corresponding homotopy does not consist of what we would normally consider systems, although this is not important for the development of robustness results. (For further discussion see [16].) It has been hypothesised that the winding number condition is equivalent to the existence of a homotopy of linear

\footnotetext{
*Received on March 12, 2002; accepted for publication on September 20, 2002.

${ }^{\dagger}$ Research School of Info. Sciences and Eng., Australian Nat. Uni., ACT 0200, Australia. E-mail: Thomas.Brinsmead@newcastle.edu.au, Phone +61-2-4921 7247, Fax: +61-2-4921 6928

${ }^{1}$ As pointed out by a reviewer, it is currently unknown [7] whether the graph topology is the weakest such that feeback stability alone is a robust property (despite an apparent statement to the contrary that appears in the Introduction of [10].)
} 
time-invariant system transfer functions, an hypothesis which is investigated in detail in [4]. In that paper, it is shown that for multivariable transfer functions, the winding number condition is equivalent to the existence of a particular multivariable transfer function homotopy. For the case of scalar transfer functions, the winding number condition is also shown to be equivalent to the existence of a particular multivariable homotopy which is achieved by embedding the scalar transfer function in a higherdimensional operator space.

The relationship between the scalar transfer function winding number condition and the existence of a scalar transfer function homotopy was not resolved in [4]. However, in [1] it was demonstrated, by a particular example, that there do exist scalar transfer functions which satisfy the winding number condition, but for which no scalar homotopy in the Vinnicombe metric between them exists. In this paper we generalise these results to develop an extra necessary and sufficient condition that two scalar transfer functions must satisfy, in addition to the winding number condition, in order for a scalar transfer function Vinnicombe metric homotopy between them to exist.

The structure of this paper is as follows. In Section 2, we review the Vinnicombe metric, define the winding number condition, and recall some properties which will be useful for the subsequent development. In the following section, we re-state the main theorem from [4] relating the winding number condition to the existence of a multivariable homotopy. We also review the main result in [1] showing that there exist some transfer functions which satisfy the winding number condition, but for which no corresponding homotopy exists. We then introduce and define a quantity which we call the "Brockett angle" corresponding to a given real number and investigate the relationship between this angle and another quantity defined for real rational functions known as the Cauchy index. We then demonstrate that the existence of a scalar homotopy between two scalar transfer functions places restrictions on the change in the Brockett angle at particular points in the transfer function. We also demonstrate why this restriction does not hold in the case of multivariable homotopies, and demonstrate the extent to which these additional necessary conditions for the existence of a scalar homotopy are also sufficient. Finally, in Section 7, we offer concluding remarks.

2. The Vinnicombe Gap Metric. For a fully detailed exposition of the Vinnicombe Gap metric, consult $[14,17]$. Here, we briefly state the definition and summarise some basic properties for discrete time plants, for which the exposition is slightly less complicated than that for continuous time plants.

In this paper a continuous time transfer function in the $s$-domain is related to a discrete transfer function in the $z$-domain by the mapping $z=\frac{1-s}{1+s}$, which maps the unstable region in the $s$-domain (the open right half plane) to the interior of the unit 
disk, and the imaginary axis to the unit circle. The origin is mapped to positive one $(+1)$, and the point at infinity is mapped to negative one $(-1)$. This contrasts with the more usual definition of a discrete time transfer function (in the $q$-domain), by the mapping $q=\frac{1+s}{1-s}$, which maps the the $s$-domain unstable region in the right half plane to the exterior of the unit disk. The reason for the somewhat unconventional choice for discrete-time mapping is in order to simplify the analysis.

Let $P_{\gamma}$ and $P_{\zeta}$ be two real rational, possibly multivariable, discrete time transfer functions with the same dimensions. Define the chordal distance between $P_{\gamma}$ and $P_{\zeta}$ at frequency $\theta$ by

$$
\begin{aligned}
\kappa\left(P_{\gamma}, P_{\zeta}, \theta\right): \stackrel{\text { def }}{=} \lim _{\hat{\theta} \rightarrow \theta} \bar{\sigma}\left\{\left[I+P_{\zeta}\left(e^{-j \hat{\theta}}\right) P_{\zeta}^{*}\left(e^{-j \hat{\theta}}\right)\right]^{-\frac{1}{2}} \times \ldots\right. \\
{\left.\left[P_{\zeta}\left(e^{-j \hat{\theta}}\right)-P_{\gamma}\left(e^{-j \hat{\theta}}\right)\right]\left[I+P_{\gamma}^{*}\left(e^{-j \hat{\theta}}\right) P_{\gamma}\left(e^{-j \hat{\theta}}\right)\right]^{-\frac{1}{2}}\right\}, }
\end{aligned}
$$

and

$$
\bar{\kappa}\left(P_{\gamma}, P_{\zeta}\right): \stackrel{\text { def }}{=} \underset{-\pi<\theta \leq \pi}{\operatorname{ess} \sup _{0}} \kappa\left(P_{\gamma}, P_{\zeta}, \theta\right) .
$$

In the definition of the chordal distance, the inverse square root $X^{-\frac{1}{2}}$ is understood to be a matrix square root of the inverse square matrix $X^{-1}$, where $X$ is positive definite Hermitian. The reason for the limit operation in the definition is to account for the possibility that either $P_{\gamma}$ or $P_{\zeta}$ has poles on the unit circle. In addition the notation $X^{*}$ represents conjugate transposition of a transfer matrix, so that $X^{*}(z)=[X(z)]^{*}$. The notation $X^{*}(z)=X(z)^{*}$ is also sometime used. For real rational transfer functions, $X^{*}(z)=X^{T}\left(z^{*}\right)$ for all $z$. Furthermore for all $|z|=1$ (that is, on the unit circle), we have that $z^{*}=z^{-1}$ so that $X^{*}(z)=X^{T}\left(z^{-1}\right)$. In the following, in order to emphasize the conjugation operation, we will usually use the notation $X^{*}(z)$ rather than $X(z)^{*}$, or $X^{T}\left(z^{*}\right)$ for real rational transfer functions, or $X^{T}\left(z^{-1}\right)$ on the unit disk, or $X\left(z^{*}\right)$, $X\left(z^{-1}\right)$ for the scalar case.

The Vinnicombe metric distance between $P_{\gamma}$ and $P_{\zeta}$ is then defined as

$$
\delta_{\nu}\left(P_{\gamma}, P_{\zeta}\right)=\bar{\kappa}\left(P_{\gamma}, P_{\zeta}\right)
$$

provided the following two conditions are satisfied:

$$
\begin{aligned}
\operatorname{det}\left[I+P_{\gamma}\left(e^{-j \theta}\right) P_{\zeta}^{*}\left(e^{j \theta}\right)\right] & \neq 0, \quad \text { for all } \theta, \\
\text { and } \quad \text { wno }\left[\operatorname{det}\left(I+P_{\gamma} P_{\zeta}^{*}\right)\right]+\breve{\eta}\left(P_{\gamma}\right)-\bar{\eta}\left(P_{\zeta}\right) & =0 .
\end{aligned}
$$

If the conditions of equations (2) and (3) are not both satisfied then $\delta_{\nu}\left(P_{\gamma}, P_{\zeta}\right)=$ 1. In the above, $P^{*}(z)$ denotes $P\left(z^{-1}\right)^{T}$, and $\operatorname{wno}(X)$ is the winding number of the transfer function $X$, that is, the number of counterclockwise encirclements of the origin by $X(z)$ as $z$ moves counter-clockwise around the unit circle (corresponding to the standard clockwise Nyquist $D$-contour in the $s$-domain) indented, if necessary, into the 
interior (that is, into the unstable region) around any unit circle poles. Finally $\breve{\eta}(X)$ and $\bar{\eta}(X)$ denote the number of poles of $X$ in the open and closed interior (unstable region) of the unit circle respectively. In this paper, we also use the notation $\breve{\mathcal{Z}}(X)$ and $(\bar{Z})(X)$ to denote the number of zeros of $X$ in the open and closed interior of the unit circle.

For scalar transfer functions $p_{\gamma}$ and $p_{\zeta}$ the defintion of the chordal distance (1) reduces to

$$
\kappa\left(p_{\gamma}, p_{\zeta}, \hat{\theta}\right)=\lim _{\theta \rightarrow \hat{\theta}} \frac{\left|p_{\zeta}\left(e^{-j \hat{\theta}}\right)-p_{\gamma}\left(e^{-j \hat{\theta}}\right)\right|}{\sqrt{\left[1+\left|p_{\zeta}\left(e^{-j \hat{\theta}}\right)\right|^{2}\right]\left[1+\left|p_{\gamma}\left(e^{-j \hat{\theta}}\right)\right|^{2}\right]}}
$$

and the determinant and winding number conditions (2) and (3) become

$$
\begin{array}{rr}
\text { for all } \theta & 1+p_{\gamma}\left(e^{-j \theta}\right) p_{\zeta}^{*}\left(e^{-j \theta}\right) \neq 0 \\
\text { and } \quad \operatorname{wno}\left[1+p_{\gamma}\left(e^{-j \theta}\right) p_{\zeta}^{*}\left(e^{-j \theta}\right)\right]+\breve{\eta}\left(p_{\gamma}\right)-\bar{\eta}\left(p_{\zeta}\right)=0 .
\end{array}
$$

An equivalent expression for the Vinnicombe metric involves normalised coprime fraction descriptions. Let $P_{\xi}=N_{\xi} M_{\xi}^{-1}=\tilde{M}_{\xi}^{-1} \tilde{N}_{\xi}$ denote normalised right and left coprime fractional descriptions [13] of $P_{\xi}$. Define

$$
\begin{aligned}
G_{\xi}^{(p+m) \times m} & =\left[\begin{array}{c}
N_{\xi} \\
M_{\xi}
\end{array}\right], \\
\text { and } \quad \tilde{G}_{\xi}^{p \times(p+m)} & =\left[\begin{array}{ll}
-\tilde{M}_{\xi} & \tilde{N}_{\xi}
\end{array}\right] .
\end{aligned}
$$

It follows that $\left[\begin{array}{cc}G_{\xi} & \tilde{G}_{\xi}^{*}\end{array}\right]$ is all-pass. The chordal distance at a frequency $\theta$ is given by

$$
\begin{aligned}
\kappa\left(P_{\gamma}, P_{\zeta}, \theta\right) & =\bar{\sigma}\left[\tilde{G}_{\zeta}\left(e^{-j \theta}\right) G_{\gamma}\left(e^{-j \theta}\right)\right], \\
& =\bar{\sigma}\left\{\tilde{M}_{\zeta}\left(e^{-j \theta}\right)\left[P_{\gamma}\left(e^{-j \theta}\right)-P_{\zeta}\left(e^{-j \theta}\right)\right] M_{\gamma}\left(e^{-j \theta}\right)\right\},
\end{aligned}
$$

where the last equality holds only for $e^{-j \theta}$ not a pole of either $P_{\gamma}$ or $P_{\zeta}$. The Vinnicombe metric may then be alternatively expressed as

$$
\delta_{\nu}\left(P_{\gamma}, P_{\zeta}\right)=\left\|\tilde{G}_{\zeta} G_{\gamma}\right\|_{\infty}=\left\|\tilde{G}_{\gamma} G_{\zeta}\right\|_{\infty}
$$

(where $\|\cdot\|_{\infty}$ represents the $\mathcal{L}_{\infty}$ norm), provided that

$$
\begin{aligned}
& \operatorname{det}\left[G_{\zeta}^{*}\left(e^{-j \theta}\right) G_{\gamma}\left(e^{-j \theta}\right)\right] \neq 0 \text { for all } \theta, \\
& \text { and } \quad \operatorname{wno}\left\{\operatorname{det}\left[G_{\zeta}^{*} G_{\gamma}\right]\right\}=0 .
\end{aligned}
$$

Otherwise $\delta_{\nu}\left(P_{\gamma}, P_{\zeta}\right)=1$. Note that conditions (2) and (9) are provably equivalent, as are conditions (3) and (10). 
Given scalar plants

$$
\begin{aligned}
& p_{\gamma}=\frac{n_{\gamma}}{m_{\gamma}}, \\
& p_{\zeta}=\frac{n_{\zeta}}{m_{\zeta}},
\end{aligned}
$$

where $\left(n_{\gamma}, m_{\gamma}\right)$ and $\left(n_{\zeta}, m_{\zeta}\right)$ are each coprime polynomial pairs, [13], let $r_{\gamma}$ and $r_{\zeta}$ be stable polynomials(that is, polynomials with no zeros on the closed unit disk) such that

$$
\begin{aligned}
r_{\gamma}^{*} r_{\gamma} & =n_{\gamma}^{*} n_{\gamma}+m_{\gamma}^{*} m_{\gamma} \\
\text { and } \quad r_{\zeta}^{*} r_{\zeta} & =n_{\zeta}^{*} n_{\zeta}+m_{\zeta}^{*} m_{\zeta}
\end{aligned}
$$

Then $p_{\gamma}$ and $p_{\zeta}$ have normalised coprime fractional descriptions

$$
\begin{gathered}
p_{\gamma}=\frac{n_{\gamma} / r_{\gamma}}{m_{\gamma} / r_{\gamma}}, \\
p_{\zeta}=\frac{n_{\zeta} / r_{\zeta}}{m_{\zeta} / r_{\zeta}},
\end{gathered}
$$

where the numerators $n_{\xi} / r_{\xi}$ and denominators $m_{\xi} / r_{\xi}$ (for $\xi=\gamma, \zeta$ ) are each rational transfer functions, which are coprime over the principal ideal domain [13] of proper stable transfer functions. We set

$$
G_{\xi}=\left[\begin{array}{c}
n_{\xi} / r_{\xi} \\
m_{\xi} / r_{\xi}
\end{array}\right] \text { and } \quad \tilde{G}_{\xi}=\left[\begin{array}{ll}
-m_{\xi} / r_{\xi} & n_{\xi} / r_{\xi}
\end{array}\right]
$$

Note that $\tilde{G}_{\xi} G_{\xi}=0$ and that $G_{\xi}^{*} G_{\xi}=1$.

That $\delta_{\nu}$ is a metric (implying that particular properties, such as the triangle inequality, for example, hold) is justified in [14].

3. Review of Previous Results. We restate a theorem from [4] regarding the relationship between the winding number condition and the existence of a homotopy between two transfer functions. Although the thereom was originally stated and proved for continuous time transfer functions, it also holds true for discrete time transfer functions.

Theorem 3.1. Let real rational $p \times m$ transfer functions $P_{\alpha}$ and $P_{\beta}$ be given, with at least one of $p$ and $m$ strictly greater than one and with $\delta_{\nu}\left(P_{\alpha}, P_{\beta}\right)<1$. Then for any given $\eta$ (which will provide an upper bound for departure from monotonicity), there exists a Vinnicombe metric homotopy, parametrised by $\lambda \in\left[\lambda_{\alpha}, \lambda_{\beta}\right]$, given by $P_{\lambda}$, varying from $P_{\alpha}$ to $P_{\beta}$ such that the following properties hold.

- Endpoint properties: $P_{\lambda}=P_{\alpha}$ for $\lambda=\lambda_{\alpha}$, and $P_{\lambda}=P_{\beta}$ for $\lambda=\lambda_{\beta}$.

- Vinnicombe Continuity Property: For every $\hat{\lambda} \in\left[\lambda_{\alpha}, \lambda_{\beta}\right]$ and $\epsilon>0$ there exists $\delta$ such that $\delta_{\nu}\left(P_{\hat{\lambda}}, P_{\lambda}\right)<\epsilon$ for all $\lambda \in\left[\lambda_{\alpha}, \lambda_{\beta}\right]$ with $|\hat{\lambda}-\lambda|<\delta$. 
- Subunitary Property: $\bar{\kappa}\left(P_{\alpha}, P_{\lambda}\right)=\sup _{\theta} \kappa\left(P_{\alpha}, P_{\lambda}, \theta\right)<1$ for all $\lambda \in\left[\lambda_{\alpha}, \lambda_{\beta}\right]$.

- Monotonicity Property (Arbitrary Closeness to): $\bar{\kappa}\left(P_{\alpha}, P_{\lambda}\right) \geq \bar{\kappa}\left(P_{\alpha}, P_{\hat{\lambda}}\right)-\eta$ for all $\hat{\lambda}, \lambda \in\left[\lambda_{\alpha}, \lambda_{\beta}\right]$ such that $\hat{\lambda} \leq \lambda$.

Conversely, if there exists a homotopy with the Endpoint and Vinnicombe Continuity properties as well as the Subunitary property, then $\delta_{\nu}\left(P_{\alpha}, P_{\beta}\right)<1$, which is equivalent to saying that if $\delta_{\nu}\left(P_{\alpha}, P_{\beta}\right)=1$ then no homotopy satisfying those three properties exists.

Proof. See [4]

Note that the continuity in the Vinnicombe metric is equivalent to continuity in the graph metric, so that the "Vinnicombe Continuity Property" could just as validly called the "Graph Metric Continuity Property", however, since our motivation is extension of the Vinnicombe metric to nonlinear systems, we retain this terminology.

Note also that the "Monotonicity Property" is not mentioned in the converse part of the theorem statement, so that there exists a homotopy connecting $P_{\alpha}$ and $P_{\beta}$ with the Endpoint, Vinnicombe Continuity and Subunitary Properties, if and only if $\delta_{\nu}\left(P_{\alpha}, P_{\beta}\right)$ is strictly less than unity. However the particular homotopy constructed for the proof does in fact possess the Monotonicity Property (in the Vinnicombe metric) so that Monotonicity is an additional property that comes "for free" and which may or may not be useful depending on the intended application of the theorem.

The situation for scalar transfer functions is a little more subtle. Of course, it is possible to embed the space of scalar transfer functions within a higher dimensional space of multivariable transfer functions. In this way it can easily be seen that the satisfaction of the winding number condition of scalar transfer functions is equivalent to the existence of a multivariable transfer function homotopy. It is shown in [1], however, that it is not equivalent to the existence of a scalar homotopy. In fact, there it is shown by counterexample, that there exist two scalar transfer functions which satisfy the winding number condition, but for which there does not exist a connecting homotopy. We present the following theorem from [1], also stated for continuous time transfer functions.

Theorem 3.2. Let $\hat{p}_{\alpha}=\frac{\epsilon}{s-1}$ and $\hat{p}_{\Omega}=-\frac{\epsilon}{s-1}$ for some $0<\epsilon<1$. Note that $\delta_{\nu}\left(\hat{p}_{\alpha}, \hat{p}_{\Omega}\right)<1$ for sufficiently small $\epsilon$. Let $u_{\alpha}(s)$ and $u_{\Omega}(s)$ be Hurwitz polynomials and let $b_{\alpha}(s)=\epsilon u_{\alpha}(s), a_{\alpha}(s)=(s-1) u_{\alpha}(s)$ and $b_{\Omega}(s)=-\epsilon u_{\Omega}(s), a_{\Omega}(s)=(s-$ $1) u_{\Omega}(s)$. Let $b_{\lambda}(s), a_{\lambda}(s)$ be homotopies on $[0,1]$ continuously linking $b_{\alpha}$ to $b_{\Omega}(s)$ and $a_{\alpha}(s)$ to $a_{\Omega}(s)$, such that setting $\hat{p}_{\lambda}=\frac{b_{\lambda}(s)}{a_{\lambda}(s)}$ gives a proper, but not necessarily strictly proper $\hat{p}_{\lambda}$. Then for some $\lambda \in(0,1)$ there holds

$$
\delta_{\nu}\left(\hat{p}_{\alpha}, \hat{p}_{\lambda}\right)=1 .
$$

Proof. See [1].

Note that if $u_{\alpha}(s)$ were not Hurwitz, then homotopies in the numerator and denominator polynomials which do not preserve the right hand plane pole-zero can- 
cellation will not translate to Vinnicombe metric homotopies of the transfer functions because the winding number condition will be violated. This is also demonstrated in [1].

The analysis in [1] proceeded first by demonstrating that no first order homotopy between two first order plants $\tilde{p}_{\alpha}=\frac{K_{\alpha}}{s+\rho_{\alpha}}$ and $\tilde{p}_{\Omega}=\frac{K_{\Omega}}{s+\rho_{\Omega}}$ exists if either $\tilde{p}_{\alpha}$ or $\tilde{p}_{\Omega}$ is unstable (that is if either $\rho_{\alpha}<0$ or $\rho_{\Omega}<0$ ) and the signs of the gains $K_{\alpha}$ and $K_{\Omega}$ are different, that is if $K_{\alpha} K_{\Omega}<0$. It was then shown, by exploiting the properties of a quantity known as the Cauchy index, defined for real rational functions, that no real rational subunitary homotopy $p_{\lambda}$ (that is, one for which $\delta_{\nu}\left(\hat{p}_{\alpha}, p_{\lambda}\right)<1$ for all $\lambda$ ) of any order exists between $\hat{p}_{\alpha}=\frac{\epsilon}{s-1}$ and $\hat{p}_{\Omega}=-\frac{\epsilon}{s-1}$, even though $\delta_{\nu}\left(\hat{p}_{\alpha}, \hat{p}_{\Omega}\right)<1$.

In the following, we generalise this result to develop an extra necessary (and sufficient) condition that two scalar transfer functions must satisfy, in addition to the winding number condition, in order for a scalar Vinnicombe metric homotopy between them to exist. However, we first introduce some concepts which will become useful in the ensuing exposition.

\section{Some Mathematical Tools:}

Cauchy indices and Brockett angles. The analysis in [1] relied on the properties of the Cauchy index. The Cauchy index is defined [9] (See in particular Chapter XV, Section 2) for a real rational function $f(s)$ over an interval $[l, u]$ of the real line (where either or both $l$ or $u$ can be at infinity) as

$$
\mathcal{I}_{l}^{u}[f(s)]=\tilde{P}_{l}^{u}[f(s)]-\tilde{N}_{l}^{u}[f(s)],
$$

where $\tilde{P}_{l}^{u}$ is the number of (positive) jumps that $f(s)$ makes from $-\infty$ to $+\infty$ as $s$ increases in the open interval $(l, u)$ and $\tilde{N}_{l}^{u}$ is the number of (negative) jumps from $+\infty$ to $-\infty$. For a rational $f(s)$ with $f(\infty)<\infty$ and denominator of degree $k$ in a coprime polynomial fraction, the Cauchy index over any interval is an integer in the set $\{-k,-k+2, \ldots, k-2, k\}$.

In [5], a slightly different perspective on the Cauchy index is developed by mapping $y$, an element of the extended real line (which includes the point at infinity) to an angle $\bar{\phi} \in(-\pi, \pi]$ by the function

$$
\bar{\phi}=\left\{\begin{array}{ccc}
2 \tan ^{-1}(y) & \text { for } & -\infty<y<\infty \\
\pi & \text { for } & y= \pm \infty
\end{array}\right.
$$

where the amibiguity in the definition of the inverse tangent is resolved by restricting it to lie within the interval $\left(-\frac{1}{2} \pi, \frac{1}{2} \pi\right]$. We shall call this angle $\bar{\phi}$, the (bounded) "Brockett angle" associated with a particular extended real number $y$.

This definition extends in a natural way to the Brockett angle corresponding to a real rational function. Consider the real rational transfer function $f(x)=\frac{b(x)}{a(x)}$, where $b(x)$ and $a(x)$ are coprime polynomials (and therefore continuous in $x$ ). Define 
a complex valued function of $x$ by

$$
\begin{aligned}
F(x) & =\frac{[1+j f(x)]^{2}}{|1+j f(x)|^{2}} \quad \text { for } x \in \mathbb{R} \\
& =\frac{1-f(x)^{2}}{1+f(x)^{2}}+2 j \frac{f(x)}{1+f(x)^{2}} \\
& =\frac{b(x)^{2}-a(x)^{2}}{b(x)^{2}+a(x)^{2}}+2 j \frac{a(x) b(x)}{b(x)^{2}+a(x)^{2}} .
\end{aligned}
$$

It is easily confirmed that for all real $f(x)$, the complex quantity $F(x)$ lies on the unit circle in the complex plane, and that the argument of the complex number $F(x)$ is the Brockett angle corresponding to the (extended) real number $f(x)$. In [5], the Cauchy index $\mathcal{I}_{\infty}^{\infty} f$ (that is, over the entire real number line) is related to the number of full rotations of the argument of $F(x)$ as $x$ varies from $-\infty$ to $+\infty$.

The Brockett angle defined in the above way is not necessarily a continuous function of $x$, since it is bounded within the interval $(-\pi, \pi]$ and it may therefore be discontinuous where $f(x)=\infty$. It is, however, possible to define a "continuous Brockett angle" over an $x$-interval $[l, u]$ corresponding to a real rational function $f(\cdot)$ by allowing the angle to range continuously beyond the bounded $(-\pi, \pi]$ interval, removing the modulo $2 \pi$ ambiguity by defining the value of the continuous Brockett angle to coincide with the bounded Brockett angle at a particular choice of $x$ in the $[l, u]$ interval. For convenience, we may take this particular choice to be the lower limit, $x=l$.

Formally, given a real rational $f(x)=\frac{b(x)}{a(x)}$, we can define a (continuous) real valued function by

$$
\begin{aligned}
\Phi(x) & =\int_{l}^{x} 2 \frac{b(t) \frac{\partial}{\partial t} a(t)-a(t) \frac{\partial}{\partial t} b(t)}{b(t)^{2}+a(t)^{2}} d t+2 \tan ^{-1} \frac{b(l)}{a(l)} \\
& =\int_{l}^{x} 2 \frac{\frac{\partial}{\partial t} f(t)}{1+f(t)^{2}} d t+2 \tan ^{-1} f(l),
\end{aligned}
$$

where as before $\tan ^{-1}: \mathbb{R} \cup \infty \rightarrow\left(-\frac{1}{2} \pi, \frac{1}{2} \pi\right]$, so that even if $a(l)=0$, then $\Phi(l)=\pi$ is

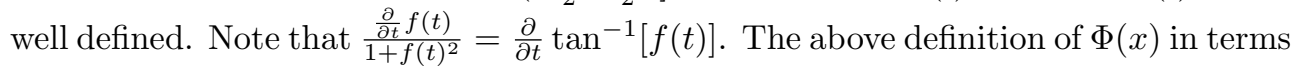
of the integral of a derivative is required to ensure that $\Phi(x)$ is continuous, even where $a(\cdot)=0$, that is $f(\cdot)=\infty$. It is fairly easy to see that $\arg [F(x)]=\bar{\phi}(x)=\Phi(x) \pm 2 n \pi$, for some integer $n$. It is also possible to let $l \rightarrow-\infty$, so that a continuous Brockett angle can be defined even for semi-infinite or doubly infinite $x$-intervals.

LEMma 4.1. Given a real rational function $f(x)=\frac{b(x)}{a(x)}$ where $b(x)$ and $a(x)$ are coprime polynomials (and therefore continuous in $x$ ), define the real function $\Phi(x)$ according to (12). The Cauchy index $\mathcal{I}_{l}^{u}$ of $f(x)$ over the interval $[l, u]$ is equal to the number of net positive strict crossings by $\Phi(x)$ of values of $\pi \pm 2 n \pi$ where $n$ is any integer, as $x$ varies over the interval $[l, u]$. 
Proof. This is fairly obvious from the definition of the Cauchy index. Consider the continuous complex function $F(x)$ defined by (11) which takes values only on the unit circle. It is easy to confirm that $\tan \left\{\frac{1}{2} \Phi(x)\right\}=\tan \left\{\frac{1}{2} \arg [F(x)]\right\}=f(x)$ and that changes as $x$ varies in the value of $f(x)$ from negative to positive infinity and vice versa correspond to changes in the argument of $F(x)$ across the angle $\pi$, that is, changes in crossings of quantity $\Phi(x)$ of values of $\pi \pm 2 n \pi$. If $\Phi(l)$ or $\Phi(u)$ is $\pi \pm 2 n \pi$, this does not count in the enumeration of strict crossings, just as the Cauchy index disregards jumps in $f(x)$ at $x=l, u$.

This lemma has related a Cauchy index of a real rational function over an interval to the change in argument of a corresponding complex function (which takes values on the unit disk) over the same real interval. It can also be seen that except in pathological cases where the continuous Brockett angle is equal to $-\pi$ (modulo $2 \pi$ ) at points $x=u$ and $x=l$, the Cauchy index $\mathcal{I}_{l}^{u} f(\cdot)$ of a function $f(\cdot)$ can be determined from knowledge of the continuous Brockett angle $\Phi(l)$ and $\Phi(u)$ at the two end points of the interval only. In fact it is fairly easy to establish that (not including pathological cases) $\mathcal{I}_{l}^{u} f(\cdot)$ is an integer such that

$$
\left|\mathcal{I}_{l}^{u} f(\cdot)-\frac{1}{2 \pi}[\Phi(u)-\Phi(l)]\right|<1 .
$$

To see this, let $\mathcal{I}_{l}^{u} f(\cdot)=N$, so that $\Phi(l)=\bar{\phi}_{l} \in(-\pi, \pi]$ and $\Phi(u)=\bar{\phi}_{u}+2 N \pi$ for some $\bar{\phi}_{u} \in(-\pi, \pi]$. then observe that

$$
\begin{aligned}
\left|\mathcal{I}_{l}^{u} f(\cdot)-\frac{1}{2 \pi}[\Phi(u)-\Phi(l)]\right| & =\left|N-\frac{1}{2 \pi}\left[\bar{\phi}_{u}+2 N \pi-\bar{\phi}_{l}\right]\right| \\
& =\left|\frac{1}{2 \pi}\left[\bar{\phi}_{u}-\bar{\phi}_{u}\right]\right|<1 .
\end{aligned}
$$

It is also possible to take care of the cases where the continuous Brockett angle is equal to $-\pi$ (modulo $2 \pi$ ) at points $x=u$ and $x=l$, by noting that for sufficiently small $\epsilon>0$ there holds

$$
\left|\mathcal{I}_{l}^{u} f(\cdot)-\frac{1}{2 \pi}[\Phi(u-\epsilon)-\Phi(l+\epsilon)]\right|<1,
$$

so that

$$
\left|\mathcal{I}_{l}^{u} f(\cdot)-\frac{1}{2 \pi}[\Phi(u)-\Phi(l)]\right| \leq 1,
$$

with equality only possible if either or both $\Phi(u)$ and $\Phi(l)$ is equal to $\pi$ modulo $2 \pi$.

4.1. Transfer function Homotopies. We now consider a homotopic family of rational functions $f_{\lambda}(x)=\frac{b_{\lambda}(x)}{a_{\lambda}(x)}$. Here $b_{\lambda}(x)$ and $a_{\lambda}(x)$ are polynomials with coefficients parametrised by $\lambda$, differentiable (with respect to $\lambda$ ) within an interval $[\alpha, \Omega]$. We can use continuity properties to show that it is possible to determine the 
Cauchy index over an interval $[l, u]$ of a function $f_{\lambda}$ as $\lambda$ varies between $[\alpha, \Omega]$ solely on the basis of knowing $f_{\alpha}$ and by observing the changes in the Brockett angle $\Phi_{\lambda}(l)$ and $\Phi_{\lambda}(u)$ at the two end points of the $x$-interval, as $\lambda$ varies.

To this end define the (continuous) change as $\lambda$ varies over an interval $[\alpha, \Omega]$ in the Brockett angle of $f_{\lambda}(x)=\frac{b_{\lambda}(x)}{a_{\lambda}(x)}$ at a point $x$ as

$$
\begin{aligned}
\Delta_{\lambda}(\alpha, \Omega, x) & =\int_{\alpha}^{\Omega} 2 \frac{b_{\lambda}(x) \frac{\partial}{\partial \lambda} a_{\lambda}(x)-a_{\lambda}(x) \frac{\partial}{\partial \lambda} b_{\lambda}(x)}{a_{\lambda}(x)^{2}+b_{\lambda}(x)^{2}} d \lambda \\
& =\int_{\alpha}^{\Omega} 2 \frac{\frac{\partial}{\partial \lambda} f_{\lambda}(x)}{1+f_{\lambda}(x)^{2}} d \lambda .
\end{aligned}
$$

Again, the above definition of $\Delta_{\lambda}(\alpha, \Omega, x)$ in terms of the integral of a derivative is required to ensure that $\Delta_{\lambda}(\alpha, \Omega, x)$ is continuous in $\Omega$. The required derivatives exist since $b_{\lambda}$ and $a_{\lambda}$ are differentiable in $\lambda$.

Conversely, in accordance with (12), we define the (continuous) change as $x$ varies over an interval $[u, l]$ in the Brockett angle of $f_{\lambda}(x)=\frac{b_{\lambda}(x)}{a_{\lambda}(x)}$ at a point $\lambda$ in the homotopy as

$$
\begin{aligned}
\Delta_{x}(l, u, \lambda) & =\int_{l}^{u} 2 \frac{b_{\lambda}(x) \frac{\partial}{\partial x} a_{\lambda}(x)-a_{\lambda}(x) \frac{\partial}{\partial x} b_{\lambda}(x)}{a_{\lambda}(x)^{2}+b_{\lambda}(x)^{2}} d x \\
& =\int_{l}^{u} 2 \frac{\frac{\partial}{\partial x} f_{\lambda}(x)}{1+f_{\lambda}(x)^{2}} d x \\
& =\Phi_{\lambda}(u)-\Phi_{\lambda}(l) .
\end{aligned}
$$

It is now a simple matter to establish that the net change in the Brockett angle over a given $x$-interval $[l, u]$ may vary with the function homotopy only as much as much as the changes in the Brockett angle over the function homotopy at the $x$-interval end points. Roughly speaking, the diagram in Figure 1 commutes. This is formalised in the following lemma.

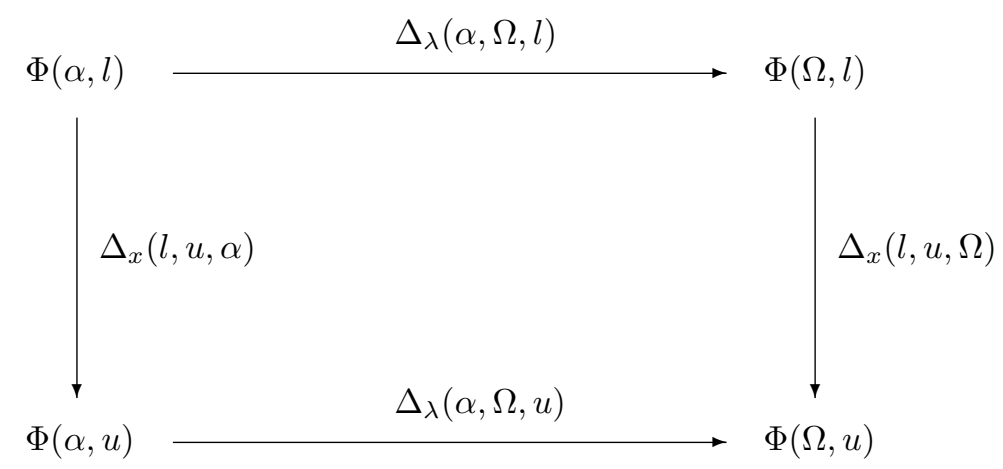

FIG. 1. Commutative Diagram 
LEMMA 4.2. For a given homotopy of real rational functions $f_{\lambda}(x)=\frac{b_{\lambda}(x)}{a_{\lambda}(x)}$ of $x$ parametrised by $\lambda$, define $\Delta_{\lambda}(\alpha, \Omega, u)$ and $\Delta_{\lambda}(\alpha, \Omega, l)$ according to equation (15) and $\Delta_{x}(l, u, \Omega)$ and $\Delta_{x}(l, u, \alpha)$ are defined according to (16). It then follows that

$$
\Delta_{x}(l, u, \Omega)-\Delta_{x}(l, u, \alpha)=\Delta_{\lambda}(\alpha, \Omega, u)-\Delta_{\lambda}(\alpha, \Omega, l) .
$$

Proof. The proof is straightforward, if somewhat involved.

$$
\begin{aligned}
\Delta_{x}(l, u, \Omega)-\Delta_{x}(l, u, \alpha) & =\int_{l}^{u} 2 \frac{\frac{\partial}{\partial x} f_{\Omega}(x)}{1+f_{\Omega}(x)^{2}} d x-\int_{l}^{u} 2 \frac{\frac{\partial}{\partial x} f_{\alpha}(x)}{1+f_{\alpha}(x)^{2}} d x \\
& =2 \int_{l}^{u} \frac{\frac{\partial}{\partial x} f_{\Omega}(x)}{1+f_{\Omega}(x)^{2}}-\frac{\frac{\partial}{\partial x} f_{\alpha}(x)}{1+f_{\alpha}(x)^{2}} d x
\end{aligned}
$$

We can express the difference in the integrand itself as an integral with respect to $\lambda$ :

$$
\begin{aligned}
\frac{\frac{\partial}{\partial x} f_{\Omega}(x)}{1+f_{\Omega}(x)^{2}}-\frac{\frac{\partial}{\partial x} f_{\alpha}(x)}{1+f_{\alpha}(x)^{2}} & =\int_{\alpha}^{\Omega} \frac{\partial}{\partial \lambda}\left[\frac{\frac{\partial}{\partial x} f_{\lambda}(x)}{1+f_{\lambda}(x)^{2}}\right] d \lambda \\
& =\int_{\alpha}^{\Omega} \frac{\left[1+f_{\lambda}(x)^{2}\right] \frac{\partial^{2}}{\partial x \partial \lambda} f_{\lambda}(x)-2 f_{\lambda}(x) \frac{\partial}{\partial \lambda} f_{\lambda}(x) \frac{\partial}{\partial x} f_{\lambda}(x)}{\left[1+f_{\lambda}(x)^{2}\right]^{2}} d \lambda .
\end{aligned}
$$

Substitution of this expression in (17) gives

$$
\begin{aligned}
& \Delta_{x}(l, u, \Omega)-\Delta_{x}(l, u, \alpha) \\
& \quad=\int_{l}^{u} \int_{\alpha}^{\Omega} \frac{\left[1+f_{\lambda}(x)^{2}\right] \frac{\partial^{2}}{\partial x \partial \lambda} f_{\lambda}(x)-2 f_{\lambda}(x) \frac{\partial}{\partial \lambda} f_{\lambda}(x) \frac{\partial}{\partial x} f_{\lambda}(x)}{\left[1+f_{\lambda}(x)^{2}\right]^{2}} d \lambda d x \\
& \quad=\int_{\alpha}^{\Omega} \int_{l}^{u} \frac{\left[1+f_{\lambda}(x)^{2}\right] \frac{\partial^{2}}{\partial x \partial \lambda} f_{\lambda}(x)-2 f_{\lambda}(x) \frac{\partial}{\partial \lambda} f_{\lambda}(x) \frac{\partial}{\partial x} f_{\lambda}(x)}{\left[1+f_{\lambda}(x)^{2}\right]^{2}} d x d \lambda .
\end{aligned}
$$

In the above, the exchange of the order of integration can be justified on the basis of the piecewise continuity of the integrand in both $x$ and $\lambda$ and the finiteness of the integral limits for $\lambda$. The inner integral can be expressed as a difference as follows.

$$
\int_{l}^{u} \frac{\left[1+f_{\lambda}(x)^{2}\right] \frac{\partial^{2}}{\partial x \partial \lambda} f_{\lambda}(x)-2 f_{\lambda}(x) \frac{\partial}{\partial \lambda} f_{\lambda}(x) \frac{\partial}{\partial x} f_{\lambda}(x)}{\left[1+f_{\lambda}(x)^{2}\right]^{2}} d x=\frac{\frac{\partial}{\partial \lambda} f_{\lambda}(u)}{1+f_{\lambda}(u)^{2}}-\frac{\frac{\partial}{\partial \lambda} f_{\lambda}(l)}{1+f_{\lambda}(l)^{2}} .
$$

Finally substituting this expression back in (18) yields

$$
\begin{aligned}
\Delta_{x}(l, u, \Omega)-\Delta_{x}(l, u, \alpha) & =\int_{\alpha}^{\Omega} \frac{\frac{\partial}{\partial \lambda} f_{\lambda}(u)}{1+f_{\lambda}(u)^{2}}-\frac{\frac{\partial}{\partial \lambda} f_{\lambda}(l)}{1+f_{\lambda}(l)^{2}} d \lambda \\
& =\Delta_{\lambda}(\alpha, \Omega, u)-\Delta_{\lambda}(\alpha, \Omega, l),
\end{aligned}
$$

as required.

The above lemma indicates that in order for the Cauchy Index over an interval $[l, u]$ of a real rational function homotopy $f_{\lambda}(x)$ to change as $\lambda$ varies from $\alpha$ to $\Omega$, the argument $\Phi_{\alpha}(x)+\Delta_{\lambda}(\alpha, \lambda, x)$ of the corresponding function $F_{\lambda}(x)$ that takes values 
on the unit disk (for all $x$ and all $\lambda$ ) must "unwind" over $\pi \pm 2 n \pi$ at one or possibly both of the interval end points $l$ and $u$ as $\lambda$ varies.

Corollary 4.3. The change in the Cauchy index $\mathcal{I}_{l}^{u} f_{\lambda}(\cdot)$, as $\lambda$ varies from $\alpha$ to $\Omega$, that is, $\mathcal{I}_{l}^{u} f_{\Omega}(\cdot)-\mathcal{I}_{l}^{u} f_{\alpha}(\cdot)$, is given by an integer such that

$$
\left|\left[\mathcal{I}_{l}^{u} f_{\Omega}(\cdot)-\mathcal{I}_{l}^{u} f_{\alpha}(\cdot)\right]-\frac{1}{2 \pi}\left[\Delta_{\lambda}(\alpha, \Omega, u)-\Delta_{\lambda}(\alpha, \Omega, l)\right]\right| \leq 2 .
$$

Proof. We investigate the change in the Cauchy index $\mathcal{I}_{l}^{u}$ as follows.

$$
\begin{aligned}
& \left|\mathcal{I}_{l}^{u} f_{\Omega}(\cdot)-\frac{1}{2 \pi} \Delta_{x}(l, u, \Omega)\right| \leq 1, \\
& \left|\mathcal{I}_{l}^{u} f_{\alpha}(\cdot)-\frac{1}{2 \pi} \Delta_{x}(l, u, \alpha)\right| \leq 1 .
\end{aligned}
$$

The above equations can be seen to be true in light of equation (14) and the fact that $\Delta_{x}(l, u, \lambda)=\Phi_{\lambda}(u)-\Phi_{\lambda}(l)$. We can therefore conclude that

$$
\begin{aligned}
\left|\mathcal{I}_{l}^{u} f_{\Omega}(\cdot)-\mathcal{I}_{l}^{u} f_{\alpha}(\cdot)-\frac{1}{2 \pi}\left[\Delta_{x}(l, u, \Omega)-\Delta_{x}(l, u, \alpha)\right]\right| & \leq 2, \\
\left|\left[\mathcal{I}_{l}^{u} f_{\Omega}(\cdot)-\mathcal{I}_{l}^{u} f_{\alpha}(\cdot)\right]-\frac{1}{2 \pi}\left[\Delta_{\lambda}(\alpha, \Omega, u)-\Delta_{\lambda}(\alpha, \Omega, l)\right]\right| & \leq 2 .
\end{aligned}
$$

This shows that if the Cauchy index $\mathcal{I}_{l}^{u} f_{\lambda}(\cdot)$ when $\lambda$ varies from $\alpha$ to $\Omega$ is to change by a value greater than two, then the argument $\Phi_{\alpha}(x)+\Delta_{\lambda}(\alpha, \lambda, x)$ of the unit disk function $F_{\lambda}(x)$ must perform a full rotation on at least one of the end points $x=l$ or $x=u$. This corresponds to the original function $f_{\lambda}(x)$ (at least at one of the end points $x=l$ or $x=u$ ) taking on all possible real values, including infinity, at least once in the homotopy.

4.2. Implications for Scalar Transfer Function Homotopies. We next establish that the existence of a Vinnicombe metric homotopy $p_{\lambda}$ between two real rational scalar transfer functions $p_{\alpha}$ and $p_{\Omega}$ places restrictions on the variation of the Brockett angle of $p_{\lambda}(z)$ at the points $z=1$ and $z=-1$, as $\lambda$ varies from $\alpha$ to $\Omega$.

We need to first show that if we have a transfer function homotopy consisting of the ratio of two polynomial homotopies, then this implies the existence of a transfer function normalised fraction description, with associated homotopies for the numberator and denominator of the fraction.

Lemma 4.4. Let $\bar{n}_{\lambda}(z), \bar{m}_{\lambda}(z)$ be two polynomial homotopies with real, bounded coefficients on $\lambda \in[\alpha, \Omega]$ continuous (differentiable) in $\lambda$ and linking $\bar{n}_{\alpha}(z)$ to $\bar{n}_{\Omega}(z)$ and $\bar{m}_{\alpha}(z)$ to $\bar{m}_{\Omega}(z)$, where for all $\lambda, \bar{n}_{\lambda}(z)$ and $\bar{m}_{\lambda}(z)$ have no common zeroes on the closed unit disk. Then there exist $n_{\lambda}(z), m_{\lambda}(z)$ two real rational scalar transfer function homotopies on $[\alpha, \Omega]$ continuous (differentiable) in $\lambda$ and linking $n_{\alpha}(z)$ to $n_{\Omega}(z)$ 
and $m_{\alpha}(z)$ to $m_{\Omega}(z)$, where $n_{\alpha}(z) m_{\alpha}(z)^{-1}=\bar{n}_{\alpha}(z) \bar{m}_{\alpha}(z)^{-1}$ and $n_{\Omega}(z) m_{\Omega}(z)^{-1}=$ $\bar{n}_{\Omega}(z) \bar{m}_{\Omega}(z)^{-1}$ and $n_{\lambda}(z), m_{\lambda}(z)$ represent the numerator and denominator of a normalised coprime fraction. That is $n_{\lambda}(z), m_{\lambda}(z)$ have no pole-zero cancellations on the closed unit disk, $n_{\lambda}^{*}(z) n_{\lambda}(z)+m_{\lambda}^{*}(z) m_{\lambda}(z)=n_{\lambda}\left(z^{-1}\right) n_{\lambda}(z)+m_{\lambda}\left(z^{-1}\right) m_{\lambda}(z)=1$ for all $z$ on the unit circle and all poles of $n_{\lambda}(z)$ and $m_{\lambda}(z)$ lie in $|z|>1$.

Proof. Let $\bar{r}_{\lambda}(z)$ be a stable spectral factorisation polynomial of $\bar{n}_{\lambda}^{*}(z) \bar{n}_{\lambda}(z)+$ $\bar{m}_{\lambda}^{*}(z) \bar{m}_{\lambda}(z)=\bar{r}_{\lambda}^{*}(z) \bar{r}_{\lambda}(z)$, that is, such that it has no zeros on the closed unit disk. Such an $\bar{r}_{\lambda}(z)$ exists because $\bar{n}_{\lambda}(z), \bar{m}_{\lambda}(z)$ are polynomials with bounded coefficients and no common zeros in the unit disk. Furthermore, since $\bar{n}_{\lambda}(z), \bar{m}_{\lambda}(z)$ are real polynomials with coefficients differentiable in $\lambda$, it follows that $\bar{r}_{\lambda}(z)$ can also be chosen so that it is a real polynomial with differentiable coefficients. If we then define

$$
\begin{aligned}
n_{\lambda}(z) & =\bar{n}_{\lambda}(z) \bar{r}_{\lambda}(z)^{-1}, \\
m_{\lambda}(z) & =\bar{m}_{\lambda}(z) \bar{r}_{\lambda}(z)^{-1},
\end{aligned}
$$

then $n_{\lambda}(z), m_{\lambda}(z)$ are two real rational scalar transfer function homotopies on $[\alpha, \Omega]$ that are differentiable in $\lambda$ and linking $n_{\alpha}(z)$ to $n_{\Omega}(z)$ and $m_{\alpha}(z)$ to $m_{\Omega}(z)$ with the required properties.

We now state the following lemma (in the $z$-domain) which asserts that the winding number condition (3) cannot be violated over a homotopy without passing through a point in the homotopy where there is a violation of the scalar version of the determinant condition (2). This is the discrete time analogue of Lemma 4.2 in [1] which applies to continuous time systems.

Lemma 4.5. Let $n_{\lambda}(z), m_{\lambda}(z)$ be two real rational stable scalar transfer function homotopies on $[\alpha, \Omega]$ continuously linking $n_{\alpha}(z)$ to $n_{\Omega}(z)$ and $m_{\alpha}(z)$ to $m_{\Omega}(z)$, where for all $\lambda$, the ratio $n_{\lambda}(z) m_{\lambda}(z)^{-1}$ is a normalised coprime fraction, implying that there are no common zeros of $n_{\lambda}(z)$ and $m_{\lambda}(z)$ on the closed unit disk and $n_{\lambda}^{*}(z) n_{\lambda}(z)+$ $m_{\lambda}^{*}(z) m_{\lambda}(z)=1$ for all $z$ on the unit circle. Then, with $p_{\alpha}=\frac{n_{\alpha}}{m_{\alpha}}, p_{\lambda}=\frac{n_{\lambda}}{m_{\lambda}}$ the two conditions

$$
\begin{aligned}
& \lim _{\lambda \rightarrow \bar{\lambda}^{-}} \delta_{\nu}\left(p_{\alpha}, p_{\lambda}\right)=1, \\
& \text { and } \quad \delta_{\nu}\left(p_{\alpha}, p_{\lambda}\right)<1 \quad \text { for all } \lambda<\bar{\lambda}
\end{aligned}
$$

will hold true if and only if $n_{\alpha}^{*} n_{\lambda}+m_{\alpha}^{*} m_{\lambda}$ is non-zero for all $z=\exp (-j \theta)$ on the unit circle with $0<\lambda<\bar{\lambda}$, and $n_{\alpha}^{*} n_{\bar{\lambda}}+m_{\alpha}^{*} m_{\bar{\lambda}}$ is zero for some $z=\exp (-j \theta)$.

Proof. It is true that

$$
\kappa\left(p_{\alpha}, p_{\lambda}, \theta\right)=\left|n_{\alpha}\left(e^{-j \theta}\right) m_{\lambda}\left(e^{-j \theta}\right)-n_{\lambda}\left(e^{-j \theta}\right) m_{\alpha}\left(e^{-j \theta}\right)\right|,
$$

and $\delta_{\nu}\left(p_{\alpha}, p_{\lambda}\right)=\underset{-\pi<\theta \leq \pi}{\operatorname{ess} \sup } \kappa^{z}\left(p_{\alpha}, p_{\lambda}, \theta\right)$ provided that

$$
\begin{aligned}
\text { for all } \theta \in(-\pi, \pi] & n_{\alpha}^{*}\left(e^{-j \theta}\right) n_{\lambda}\left(e^{-j \theta}\right)+m_{\alpha}^{*}\left(e^{-j \theta}\right) m_{\lambda}\left(e^{-j \theta}\right) \neq 0, \\
& \text { and } \quad \operatorname{wno}\left[n_{\alpha}(\cdot)^{*} n_{\lambda}(\cdot)+m_{\alpha}(\cdot)^{*} m_{\lambda}(\cdot)\right]=0 .
\end{aligned}
$$


One can verify that for all $\theta$

$$
\begin{aligned}
& \quad\left|n_{\alpha}\left(e^{-j \theta}\right) m_{\lambda}\left(e^{-j \theta}\right)-n_{\lambda}\left(e^{-j \theta}\right) m_{\alpha}\left(e^{-j \theta}\right)\right|^{2} \\
& \quad+\left|n_{\alpha}^{*}\left(e^{-j \theta}\right) n_{\lambda}\left(e^{-j \theta}\right)+m_{\alpha}^{*}\left(e^{-j \theta}\right) m_{\lambda}\left(e^{-j \theta}\right)\right|^{2}=1 .
\end{aligned}
$$

When $\lambda<\bar{\lambda}$ the condition $\delta_{\nu}\left(p_{\alpha}, p_{\lambda}\right)<1$ implies that the winding number condition holds and therefore $\left|n_{\alpha}^{*}\left(e^{-j \theta}\right) n_{\lambda}\left(e^{-j \theta}\right)+m_{\alpha}^{*}\left(e^{-j \theta}\right) m_{\lambda}\left(e^{-j \theta}\right)\right| \neq 0$ for all $\theta$.

When $\lambda \rightarrow \bar{\lambda}^{-}$we have that $\delta_{\nu}\left(p_{\alpha}, p_{\lambda}\right) \rightarrow 1$. This can only be because either $\kappa\left(p_{\alpha}, p_{\lambda}, \theta\right) \rightarrow 1$ at some $\theta$, that is, in light of equation (20), that $\left|n_{\alpha}\left(e^{-j \theta}\right) m_{\lambda}\left(e^{-j \theta}\right)-n_{\lambda}\left(e^{-j \theta}\right) m_{\alpha}\left(e^{-j \theta}\right)\right| \rightarrow 1$; or the winding number condition (21) fails so that $\left|n_{\alpha}^{*}\left(e^{-j \theta}\right) n_{\lambda}\left(e^{-j \theta}\right)+m_{\alpha}^{*}\left(e^{-j \theta}\right) m_{\lambda}\left(e^{-j \theta}\right)\right| \rightarrow 0$, or indeed both. (The winding number cannot change without $n_{\alpha}^{*}\left(e^{-j \theta}\right) n_{\lambda}\left(e^{-j \theta}\right)+m_{\alpha}^{*}\left(e^{-j \theta}\right) m_{\lambda}\left(e^{-j \theta}\right)$ having a zero pass across the boundary of the unit disk). Inspection of equation (22) shows that both conditions causing $\delta_{\nu}\left(p_{\alpha}, p_{\lambda}\right)=1$ will necessarily operate simultaneously, at the one frequency. Similar reasoning will demonstrate the reverse implication.

We now define a change in Brockett angle corresponding to the value of $p_{\lambda}(z)$ at a fixed real value of $z$ as $\lambda$ varies as

$$
\Delta_{\lambda}(\alpha, \mu, z)=\int_{\alpha}^{\mu} 2 \frac{\frac{\partial}{\partial \lambda} p_{\lambda}(z)}{1+p_{\lambda}(z)^{2}} d \lambda
$$

and a continuous Brockett angle as $\lambda$ varies as

$$
\Phi_{\lambda}(z)=2 \tan ^{-1} p_{\alpha}(z)+\Delta_{\lambda}(\alpha, \lambda, z) .
$$

Note that the modulo $2 \pi$ ambiguity is removed by specifying the value of $\Phi_{\lambda}(z)$ at $\lambda=\alpha$. (As an aside, note that, strictly speaking, this definition may agree with that presented in equation (12), only up to modulo $2 \pi$. In equation (12) the modulo $2 \pi$ ambiguity in defining $\Phi$ was resolved by requiring $\Phi(l)$ to lie in the interval $(-\pi, \pi]$, whereas in equation (24), it is resolved by specifying $\Phi_{\alpha}(z)$. This issue does not affect the correctness of the following development presented here.) Clearly, $\tan \frac{1}{2} \Phi_{\lambda}(z)=$ $p_{\lambda}(z)=\frac{n_{\lambda}(z)}{m_{\lambda}(z)}$ for real $z$. Also, because $\frac{n_{\lambda}(z)}{m_{\lambda}(z)}$ is normalised, that is, $\left|n_{\lambda}(z)\right|^{2}+$ $\left|m_{\lambda}(z)\right|^{2}=1$ on the unit circle, it follows that at the points $z=1$ and $z=-1$ we have either $n_{\lambda}(z)=\sin \left[\frac{1}{2} \Phi_{\lambda}(z)\right]$ and $m_{\lambda}(z)=\cos \left[\frac{1}{2} \Phi_{\lambda}(z)\right]$, or $n_{\lambda}(z)=-\sin \left[\frac{1}{2} \Phi_{\lambda}(z)\right]$ and $m_{\lambda}(z)=-\cos \left[\frac{1}{2} \Phi_{\lambda}(z)\right]$. We can also show, because $n_{\lambda}, m_{\lambda}$ are real rational, that

$$
\begin{aligned}
n_{\alpha}^{*}(1) n_{\lambda}(1)+m_{\alpha}^{*}(1) m_{\lambda}(1) & =n_{\alpha}(1) n_{\lambda}(1)+m_{\alpha}(1) m_{\lambda}(1) \\
& = \pm\left\{\cos \left[\frac{1}{2} \Phi_{\alpha}(1)\right] \cos \left[\frac{1}{2} \Phi_{\lambda}(1)\right]+\sin \left[\frac{1}{2} \Phi_{\alpha}(1)\right] \sin \left[\frac{1}{2} \Phi_{\lambda}(1)\right]\right\} \\
& = \pm \cos \left[\frac{1}{2} \Phi_{\lambda}(1)-\frac{1}{2} \Phi_{\alpha}(1)\right] \\
& = \pm \cos \left[\frac{1}{2} \Delta_{\lambda}(\alpha, \lambda, 1)\right] .
\end{aligned}
$$


Similarly

$$
\begin{aligned}
n_{\alpha}^{*}(-1) n_{\lambda}(-1)+m_{\alpha}^{*}(-1) m_{\lambda}(-1) & = \pm \cos \left[\frac{1}{2} \Phi_{\lambda}(-1)-\frac{1}{2} \Phi_{\alpha}(-1)\right] \\
& = \pm \cos \left[\frac{1}{2} \Delta_{\lambda}(\alpha, \lambda,-1)\right]
\end{aligned}
$$

This allows us to conclude the following.

Lemma 4.6. If $p_{\lambda}$ is a Vinnicombe metric homotopy of real rational transfer functions for $\lambda \in[\alpha, \Omega]$ (piecewise differentiable in $\lambda$ ), then the change in the Brockett angle $\Delta_{\lambda}(\alpha, \lambda, z)$ defined by equation (23) at the points $z=1$ and $z=-1$ is restricted to the interval $(-\pi, \pi)$ for all $\lambda$.

Proof. By Lemma 4.5, the winding number condition, equation (21) being true for all $\lambda$ implies that as $\lambda$ varies, $\cos \left[\frac{1}{2} \Delta_{\lambda}(\alpha, \lambda, \pm 1)\right] \neq 0$ that is $\Delta_{\lambda}(\alpha, \lambda, \pm 1)$ is in the interval $(-\pi, \pi)$ for all $\lambda$.

At the points $z=1$ and $z=-1$ the quantity $p_{\lambda}(z)$ is real. Lemma 4.6 implies that a necessary condition for the winding number condition to hold throughout a $p_{\lambda}$ homotopy, is that at the particular frequencies $z= \pm 1$ (at DC and the folding frequency respectively) the net change as $\lambda$ varies, in the corresponding angle may not be $\pi$. This is equivalent to saying that at no value of $\lambda$ is it permitted for $p_{\lambda}(1)=-p_{\alpha}^{-1}(1)$ or $p_{\lambda}(-1)=-p_{\alpha}^{-1}(-1)$.

It then follows from Lemma 4.2 that if there exists a homotopy from $p_{\alpha}$ to $p_{\Omega}$, then the quantity

$$
\Delta_{x}(-1,1, \Omega)-\Delta_{x}(-1,1, \alpha)=\Delta_{\lambda}(\alpha, \Omega, 1)-\Delta_{\lambda}(\alpha, \Omega,-1)
$$

is restricted in allowable possible values. That is, change $\Delta_{x}(-1,1, \lambda)$ in the Brockett angle over the $x$-interval $[-1,1]$ may not vary by too much as $\lambda$ varies from $\alpha$ to $\Omega$. Specifically, since $\Delta_{\lambda}(\alpha, \Omega, 1) \in(-\pi, \pi)$ and $\Delta(\alpha, \Omega,-1) \in(-\pi, \pi)$ if the winding number condition is satisfied for all $\lambda$, then $\Delta_{x}(-1,1, \Omega)-\Delta(-1,1, \alpha) \in(-2 \pi, 2 \pi)$. We formalise this is the following corollary, which is one of the main results of this paper.

Corollary 4.7 (Main Result). Assume that $p_{\lambda}(z)=n_{\lambda}(z) m_{\lambda}(z)^{-1}$ (for $\lambda \in$ $[\alpha, \Omega])$ is a homotopy of real rational transfer functions where $n_{\lambda}(z)$ and $m_{\lambda}(z)$ form a real rational normalised stable coprime factorisation and the winding number condition $\cos \left[\frac{1}{2} \Delta_{\lambda}(\alpha, \lambda, z)\right] \neq 0$ holds at $z=-1$ and $z=1$. Then the net change in the argument at $z=1$, that is, $\Delta_{\lambda}(\alpha, \lambda, 1)$ is bounded within the interval $(-\pi, \pi)$ for all $\lambda$. Similarly, the net change in the argument at $z=-1$, that is, $\Delta_{\lambda}(\alpha, \lambda,-1)$ is also bounded for all $\lambda$.

Furthermore, if $\mathcal{I}_{-1}^{1} p_{\lambda}(z)-\mathcal{I}_{-1}^{1} p_{\alpha}(z)=N$, then $N \in\{-2,-1,0,1,2\}$.

Proof. This is a corollary Lemma 4.6, using Corollary 4.3.

Since $p_{\lambda}(z)$ is a homotopy of real rational transfer functions such that the winding number condition $\cos \left[\frac{1}{2} \Delta_{\lambda}(\alpha, \lambda, z)\right] \neq 0$ holds at $z=-1$ and $z=1$, it follows by 
Lemma 4.6 that both $\Delta_{\lambda}(\alpha, \lambda, 1) \in(-\pi, \pi)$ and $\Delta_{\lambda}(\alpha, \lambda,-1) \in(-\pi, \pi)$. Hence $\Delta_{\lambda}(\alpha, \lambda, 1)-\Delta_{\lambda}(\alpha, \lambda, 1) \in(-2 \pi, 2 \pi)$.

Then, by Corollary 4.3 we have

$$
\left|\left[\mathcal{I}_{-1}^{1} p_{\lambda}(z)-\mathcal{I}_{-1}^{1} p_{\alpha}(z)\right]-\frac{1}{2 \pi}\left[\Delta_{\lambda}(\alpha, \lambda, 1)-\Delta_{\lambda}(\alpha, \lambda, 1)\right]\right| \leq 2 .
$$

Since $\left|\frac{1}{2 \pi}\left[\Delta_{\lambda}(\alpha, \lambda, 1)-\Delta_{\lambda}(\alpha, \lambda, 1)\right]\right|<1$ it follows that $\mathcal{I}_{-1}^{1} p_{\lambda}(z)-\mathcal{I}_{-1}^{1} p_{\alpha}(z) \in(3,3)$ but since $\mathcal{I}_{-1}^{1} p_{\lambda}(z)-\mathcal{I}_{-1}^{1} p_{\alpha}(z)=N$ is an integer then $N \in\{-2,-1,0,1,2\}$.

REMARK 4.8. In the course of letting $\lambda$ vary for a Vinnicombe homotopy $p_{\lambda}$, the Cauchy index $\mathcal{I}_{-1}^{1} p_{\lambda}(z)$ cannot change by more than two, since this would force the value of $p_{\lambda}(z)$ at at least one of either $z=1$ or $z=-1$ to take on all possible real values, including the forbidden value $p_{\lambda}(1)=-p_{\alpha}^{-1}(1)$ or $p_{\lambda}(-1)=-p_{\alpha}^{-1}(-1)$, leading to a violation of the winding number condition, which requires that $1+p_{\lambda}\left(z^{-1}\right)^{*} p_{\alpha}(z) \neq$ 0 , for all $z$ on the unit disk.

5. Revisiting a Previous Example. Our main result, Corollary 4.7 has indicated that if a Vinnicombe metric homotopy exists, then there is a restriction on the allowable change in the Cauchy index over the interval $[-1,1]$. Armed with this this insight, we can take a fresh look at the example presented in [1].

We first note that the Cauchy indices of $\epsilon /(s-1)$ and $-\epsilon /(s-1)$ are minus one and plus one respectively. They only differ by two, which is not forbidden by Corollary 4.7. However, by using the tools presented in previous sections, we can show that there does not exist a homotopy from $\epsilon /(s-1)$ to $-\epsilon /(s-1)$.

In order to see this, consider the restriction on the change in Brockett angle at $s=0$ and $s=\infty$ (corresponding for continuous systems to $z=+1$ and $z=-1$ in discrete time). We recall that the Brockett angle at frequencies $s=0$ and $s=\infty$ at the end points $\lambda=\alpha$ and $\lambda=\Omega$ of the homotopy are specified modulo $2 \pi$ by the value of $p_{\lambda}(z)$. However, the net change in the argument as $\lambda$ varies limited to $(-\pi, \pi)$. Taken together, these three facts specify the change in $\Delta_{x}(0, \infty, \lambda)$ as $\lambda$ varies.

It is fairly easy to see that for the plant $\epsilon /(s-1)$

$$
\begin{aligned}
\Phi_{\alpha}(0) & =2 \tan ^{-1}-\epsilon \approx-2 \epsilon, \\
\Phi_{\alpha}(\infty) & =-2 \pi \\
\Delta_{x}(0, \infty, \alpha) & \approx-2(\pi-\epsilon)
\end{aligned}
$$

and that for $-\epsilon /(s-1)$

$$
\begin{aligned}
\Phi_{\Omega}(0) & =2 \tan ^{-1} \epsilon \approx 2 \epsilon, \\
\Phi_{\Omega}(\infty) & =2 \pi, \\
\Delta_{x}(0, \infty, \alpha) & \approx 2(\pi-\epsilon) .
\end{aligned}
$$

It also follows that since $\Phi_{\alpha}(0) \approx-2 \epsilon$, that $\Phi_{\Omega}(0) \approx 2 \epsilon$ and $\Delta_{\lambda}(\alpha, \Omega, 0)=\Phi_{\Omega}(0)-$ $\Phi_{\alpha}(0)+2 n \pi \approx 4 \epsilon+2 n \pi$. However, if a homotopy exists that does not violate the 
winding number condition then $\Delta_{\lambda}(\alpha, \Omega, 0) \in(-\pi, \pi)$ so that $\Delta_{\lambda}(\alpha, \Omega, 0) \approx 4 \epsilon$. Similarly $\Delta_{\lambda}(\alpha, \Omega, \infty)=\Phi_{\Omega}(0)-\Phi_{\alpha}(0)+2 n \pi=4 \pi+2 n \pi$ and since $\Delta_{\lambda}(\alpha, \Omega, \infty) \in(-\pi, \pi)$ then $\Delta_{\lambda}(\alpha, \Omega, \infty)=0$. From this we can determine that if a homotopy exists, then the winding number condition restriction on the change as $\lambda$ varies in the continuous Brockett angle at the end points of the $s$-interval at $s=0$ and $s=\infty$ determines $\Delta_{\lambda}(\alpha, \Omega, \infty)-\Delta_{\lambda}(\alpha, \Omega, 0) \approx-4 \epsilon$.

If we now look at the change as $x$ varies in the continuous Brockett angle at the end points of the $\lambda$-interval, at $\lambda=\alpha$ and $\lambda=\Omega$, we see that $\Delta_{x}(0, \infty, \Omega)-\Delta_{x}(0, \infty, \alpha) \approx$ $-4 \epsilon+4 \pi \neq-4 \epsilon$. However, this leads to a contradiction with Lemma 4.2.

The changes of the Cauchy index alone do not tell the whole story and are insufficient to establish the existence or non-existence of a homotopy. The reason for this is that the Cauchy index is related to changes in the absolute Brockett angle (modulo $2 \pi$ ), but the winding number condition only places restrictions on the relative changes as $\lambda$ varies in the (continuous) Brockett angle at the end points of the $x$-interval.

5.1. The Cauchy Index may Change by Two during a Homotopy. We have just seen that there exist transfer functions which have Cauchy indices that differ by two, and yet for which there exists no connecting Vinnicombe metric homotopy. In order to demonstrate that the conclusions in Corollary 4.7 are not conservative, we present a Vinnicombe metric homotopy which does involve a Cauchy index change of two.

Define a variable parameter $\beta_{\lambda} \in[1-\epsilon, 1+\epsilon]$ where $\epsilon>0$ is a small positive real number. The parameter $\beta_{\lambda}$ is presumed to vary with $\lambda \in[\alpha, \Omega]$ from $\beta_{\alpha}=1-\epsilon$ to $\beta_{\Omega}=1+\epsilon$

Define a variable gain $K_{\lambda}$ by

$$
K_{\lambda}=\sqrt{\left(16+\frac{1}{16}\right) \beta_{\lambda}^{2}-\left(1+\beta_{\lambda}^{4}\right)} \in \approx 3.75 \pm 15 \epsilon,
$$

and a homotopy of transfer functions by

$$
\begin{aligned}
p_{\lambda} & =\frac{K_{\lambda}}{z\left(z+\beta_{\lambda}\right)\left(z-\beta_{\lambda}\right)} \\
& =\frac{K_{\lambda}}{2 \beta_{\lambda}^{2}}\left(\frac{-2}{z}+\frac{1}{z+\beta_{\lambda}}+\frac{1}{z-\beta_{\lambda}}\right) .
\end{aligned}
$$

The reason that a non-constant gain has been chosen is in order to make the expressions for the normalised coprime fraction description of $p_{\lambda}$ simpler: in fact, for small $\epsilon$, the gain $K_{\lambda}$ does not vary particularly much with $\lambda$. A normalised fraction description of $p_{\lambda}$ is given by

$$
G_{\lambda}=\left[\begin{array}{c}
\frac{K_{\lambda}}{\beta_{\lambda}\left(\frac{1}{2} z+2\right)\left(\frac{1}{2} z-2\right)} \\
\frac{z\left(z+\beta_{\lambda}\right)\left(z-\beta_{\lambda}\right)}{\beta_{\lambda}\left(\frac{1}{2} z+2\right)\left(\frac{1}{2} z-2\right)}
\end{array}\right] .
$$


To check that the above coprime fraction description is normalised, it is a simple matter to confirm that

$$
K_{\lambda}^{2}+\left(z^{2}-\beta_{\lambda}^{2}\right)\left(z^{-2}-\beta_{\lambda}^{2}\right)=\beta_{\lambda}^{2}\left(\frac{1}{4} z^{2}-4\right)\left(\frac{1}{4} z^{-2}-4\right) .
$$

It follows easily that

$$
\begin{aligned}
\tilde{G}_{\lambda} & =\left[\begin{array}{ll}
-\frac{z\left(z+\beta_{\lambda}\right)\left(z-\beta_{\lambda}\right)}{\beta_{\lambda}\left(\frac{1}{2} z+2\right)\left(\frac{1}{2} z-2\right)} & \frac{K_{\lambda}}{\beta_{\lambda}\left(\frac{1}{2} z+2\right)\left(\frac{1}{2} z-2\right)}
\end{array}\right], \\
G_{\alpha} & =\left[\begin{array}{ll}
\frac{K_{\alpha}}{\beta_{\alpha}\left(\frac{1}{2} z+2\right)\left(\frac{1}{2} z-2\right)} \\
\frac{z\left(z+\beta_{\alpha}\right)\left(z-\beta_{\alpha}\right)}{\beta_{\alpha}\left(\frac{1}{2} z+2\right)\left(\frac{1}{2} z-2\right)}
\end{array}\right], \\
\tilde{G}_{\alpha} & =\left[\begin{array}{ll}
-\frac{z\left(z+\beta_{\alpha}\right)\left(z-\beta_{\alpha}\right)}{\beta_{\alpha}\left(\frac{1}{2} z+2\right)\left(\frac{1}{2} z-2\right)} & \frac{K_{\alpha}}{\beta_{\alpha}\left(\frac{1}{2} z+2\right)\left(\frac{1}{2} z-2\right)}
\end{array}\right] .
\end{aligned}
$$

We now look at the Vinnicombe distance.

$$
\begin{aligned}
\tilde{G}_{\alpha} G_{\lambda} & =\frac{z\left[K_{\lambda} \beta_{\alpha}\left(z^{2}-\lambda_{\alpha}^{4}\right)-K_{\alpha} \beta_{\lambda}\left(z^{2}-\beta_{\lambda}^{2}\right)\right]}{\beta_{\alpha} \beta_{\lambda}\left(\frac{1}{2} z+2\right)\left(\frac{1}{2} z-2\right)} . \\
\left\|\tilde{G}_{\alpha} G_{\lambda}\right\|_{\infty} & <\frac{4}{9(1-\epsilon)^{4}}\left|K_{\lambda} \beta_{\alpha}\left(z^{2}-\lambda_{\alpha}^{4}\right)-K_{\alpha} \beta_{\lambda}\left(z^{2}-\beta_{\lambda}^{2}\right)\right| \\
& <\frac{4}{9(1-\epsilon)^{4}}\left(\left|K_{\lambda} \beta_{\alpha}-K_{\alpha} \beta_{\lambda}\right|+\left|K_{\lambda} \beta_{\alpha}^{3}-K_{\alpha} \beta_{\lambda}^{3}\right|\right) \\
& \approx \mathcal{O}(\epsilon) .
\end{aligned}
$$

Since (for small $\epsilon$ ) the quantity $\left\|\tilde{G}_{\alpha} G_{\lambda}\right\|_{\infty}$ remains small, and never approaches the value unity as $\lambda$ varies it is established that $p_{\lambda}$ is a Vinnicombe metric homotopy such that $\delta_{\nu}\left(p_{\alpha}, p_{\lambda}\right)<1$ for all $\lambda \in[\lambda, \Omega]$.

Note that the Cauchy index over a real interval $[l, u]$ of a real rational transfer function $f(x)$ is equal to the number of positive signed residuals of real poles of $f(x)$ in the interval $[l, u]$, minus the number of negative signed residuals of real poles of $f(x)$ in the interval. From the partial fraction expansion of $p_{\lambda}$, it is possible to see that the Cauchy index within the interval $[-1,1]$ differs by 2 as $\beta_{\lambda}$ varies from just smaller than unity to just greater than unity.

This demonstrates that it is possible for the Cauchy index over the interval $[-1,1]$ to vary by up to two in a scalar Vinnicombe metric homotopy.

6. Scalar Vinnicombe Metric Homotopies: Necessary and Sufficient Conditions for Existence. In this section we develop necessary and sufficient conditions for the existence of a Vinnicombe metric homotopy between two given scalar transfer functions. However, in order to simplify the analysis, we present a number of lemmas which allow us to consider, without loss of generality, one end point of the homotopy to be fixed at unity over all frequencies.

We first define a linear transformation on transfer function space that maps an arbitrary given transfer function $p_{\alpha}$ to 1 . Provided $p_{\alpha}$ possesses no unit circle poles, 
the transformation preserves certain properties that are desirable from the point of view of Vinnicombe metric analysis.

Lemma 6.1. Consider the linear transformation $T: \mathbb{R}(s) \rightarrow \mathbb{R}(s)$, parametrised by $p_{\alpha} \in \mathbb{R}(s)$.

$$
T\left(p_{\lambda}\right)=\frac{2 p_{\alpha}^{*}}{1+\left|p_{\alpha}\right|^{2}} p_{\lambda}+\frac{1-\left|p_{\alpha}\right|^{2}}{1+\left|p_{\alpha}\right|^{2}}
$$

Then $T\left(p_{\alpha}\right)=1$, and furthermore $T\left(p_{\lambda}\right)\left[T\left(p_{\alpha}\right)\right]^{*}+1=0$ if and only if $p_{\lambda} p_{\alpha}^{*}+1=0$. In addition, if normalised coprime fraction descriptions of $p_{\alpha}, p_{\lambda}, \bar{p}_{\alpha}=T\left(p_{\alpha}\right)$ and $\bar{p}_{\lambda}=T\left(p_{\lambda}\right)$ respectively by $\frac{n_{\alpha}}{m_{\alpha}}, \frac{n_{\lambda}}{m_{\lambda}}, \frac{\bar{n}_{\alpha}}{\bar{m}_{\alpha}}$ and $\frac{\bar{n}_{\lambda}}{\bar{m}_{\lambda}}$ then provided $p_{\alpha}$ has no poles or zeroes on the unit circle

$$
n_{\alpha}^{*}\left(e^{-j \theta}\right) n_{\lambda}\left(e^{-j \theta}\right)+m_{\alpha}^{*}\left(e^{-j \theta}\right) m_{\lambda}\left(e^{-j \theta}\right)=0
$$

for some $\theta \in(-\pi, \pi]$ if and only if

$$
\bar{n}_{\alpha}^{*}\left(e^{-j \theta}\right) \bar{n}_{\lambda}\left(e^{-j \theta}\right)+\bar{m}_{\alpha}^{*}\left(e^{-j \theta}\right) \bar{m}_{\lambda}\left(e^{-j \theta}\right)=0 .
$$

If $p_{\alpha}$ has no poles on the unit circle then (27) implies (26), but the converse does not necessarily hold. If $p_{\alpha}$ has no zeroes on the unit circle then (26) implies (27), but the converse does not necessarily hold.

Proof. That $T\left(p_{\alpha}\right)=1$ follows immediately from the definition of $T$.

Provided that $p_{\alpha}$ is finite, it is also fairly easy to see that

$$
T\left(p_{\lambda}\right)\left[T\left(p_{\alpha}\right)\right]^{*}+1=0
$$

implies

$$
\begin{aligned}
p_{\alpha}^{*} p_{\lambda}+1-\left|p_{\alpha}\right|^{2}+1+\left|p_{\alpha}\right|^{2} & =0, \\
p_{\alpha}^{*} p_{\lambda}+1 & =0,
\end{aligned}
$$

and vice versa.

At poles of $p_{\alpha}$ if we know that $p_{\alpha}^{*} p_{\lambda}+1=0$, then we can conclude that $p_{\lambda}=0$ at that point and hence from equation $(25)$ that $T\left(p_{\lambda}\right)=-1$, that is $T\left(p_{\lambda}\right)\left[T\left(p_{\alpha}\right)\right]^{*}+1=$ 0 . Hence $p_{\alpha}^{*} p_{\lambda}+1=0$ implies $T\left(p_{\lambda}\right)\left[T\left(p_{\alpha}\right)\right]^{*}+1=0$ and, everywhere except for poles of $p_{\alpha}$ it is true that $T\left(p_{\lambda}\right)\left[T\left(p_{\alpha}\right)\right]^{*}+1=0$ implies $p_{\alpha}^{*} p_{\lambda}+1=0$.

Note also that

$$
\bar{n}_{\alpha}(z)=\bar{m}_{\alpha}(z)=\frac{1}{\sqrt{2}}
$$

for all $z$ and hence equation (27) is equivalent to $\bar{n}_{\lambda}\left(e^{-j \theta}\right)+\bar{m}_{\lambda}\left(e^{-j \theta}\right)=0$ and also to $T\left[p_{\lambda}\left(e^{-j \theta}\right)\right]=-1$. Since $T\left(p_{\alpha}\right)^{*}=1$ for all $z$, this is also equivalent to $T\left(p_{\alpha}\right)^{*} T\left(p_{\lambda}\right)+1=0$. Provided that that $e^{-j \theta}$ is not a pole of $p_{\alpha}$ then we can also conclude that $p_{\alpha}^{*} p_{\lambda}+1=0$. 
For the foward implication, that is to show that equation (26) implies equation (27) we need to assume that $n_{\alpha}\left(e^{-j \theta}\right) \neq 0$. We now consider separate cases for $p_{\alpha}\left(e^{-j \theta}\right)$.

Case 1 (neither a pole nor a zero of $p_{\alpha}$ ): We first consider the case where both $n_{\alpha}\left(e^{-j \theta}\right) \neq 0$ and $m_{\alpha}\left(e^{-j \theta}\right) \neq 0$. If $n_{\lambda}\left(e^{-j \theta}\right)=0$ then equation (26) implies that $m_{\lambda}\left(e^{-j \theta}\right)=0$, which contradicts that $n_{\lambda}(z)$ and $m_{\lambda}(z)$, have no common zeros in the closed unit disk. Similarly if $m_{\lambda}\left(e^{-j \theta}\right)=0$ then equation (26) implies that $n_{\lambda}\left(e^{-j \theta}\right)=0$, which is also a contradiction. Hence both $n_{\lambda}\left(e^{-j \theta}\right) \neq 0$ and $m_{\lambda}\left(e^{-j \theta}\right) \neq 0$ and thus both $p_{\alpha}$ and $p_{\lambda}$ are finite and non-zero at $z=e^{-j \theta}$. It follows that (26), taken together with the facts $m_{\alpha}\left(e^{-j \theta}\right) \neq 0$ and $m_{\lambda}\left(e^{-j \theta}\right) \neq 0$, implies

$$
\frac{n_{\alpha}^{*}\left(e^{-j \theta}\right)}{m_{\alpha}^{*}\left(e^{-j \theta}\right)} \frac{n_{\lambda}\left(e^{-j \theta}\right)}{m_{\lambda}\left(e^{-j \theta}\right)}+1=0,
$$

that is,

$$
p_{\alpha}^{*}\left(e^{-j \theta}\right) p_{\lambda}\left(e^{-j \theta}\right)+1=0 .
$$

We recall that since $e^{-j \theta}$ is not a pole of $p_{\alpha}$, then $p_{\alpha}^{*}\left(e^{-j \theta}\right) p_{\lambda}\left(e^{-j \theta}\right)+1=0$ implies $T\left[p_{\lambda}\left(e^{-j \theta}\right)\right]=-1$ which is equivalent to $(27)$.

Case 2 (at a pole): On the other hand, if $m_{\alpha}\left(e^{-j \theta}\right)=0$, then taking equation (26) as given, we conclude that $n_{\lambda}\left(e^{-j \theta}\right)=0$ and by the coprimeness of $n_{\lambda}(z)$ and $m_{\lambda}(z)$ we have $m_{\lambda}\left(e^{-j \theta}\right) \neq 0$. Thus $m_{\alpha}\left(e^{-j \theta}\right)=0$ and $n_{\lambda}\left(e^{-j \theta}\right)=0$. From equation (25) we have that $T\left(p_{\lambda}\left(e^{-j \theta}\right)\right)=-1$ which is equivalent to $(27)$.

For the converse, that is to show that equation (27) implies equation (26) we need to assume that there are no poles of $p_{\alpha}$ on the unit disk. Recall that equation (27) is equivalent to $T\left(p_{\lambda}\left(e^{-j \theta}\right)\right)=-1$ which, in light of the assumption of no unit disk poles, also implies that $p_{\alpha}^{*}\left(e^{-j \theta}\right) p_{\lambda}\left(e^{-j \theta}\right)=-1$. We now consider separate cases for $p_{\alpha}\left(e^{-j \theta}\right)$.

Case 1 (neither a pole nor zero of $\left.p_{\alpha}\right)$ : We first assume that both $n_{\alpha}\left(e^{j \theta}\right) \neq 0$ and $m_{\alpha}\left(e^{j \theta}\right) \neq 0$. Since $e^{-j \theta}$ is not a pole of $p_{\alpha}$, then $p_{\alpha}^{*}\left(e^{-j \theta}\right) p_{\lambda}\left(e^{-j \theta}\right)=-1$. It then follows that both $n_{\lambda}\left(e^{-j \theta}\right) \neq 0$ and $m_{\lambda}\left(e^{-j \theta}\right) \neq 0$. We can also write

$$
\begin{aligned}
\frac{n_{\alpha}^{*}\left(e^{-j \theta}\right)}{m_{\alpha}^{*}\left(e^{-j \theta}\right)} \frac{n_{\lambda}\left(e^{-j \theta}\right)}{m_{\lambda}\left(e^{-j \theta}\right)}+1 & =0, \\
n_{\alpha}^{*}\left(e^{-j \theta}\right) n_{\lambda}\left(e^{-j \theta}\right)+m_{\alpha}^{*}\left(e^{-j \theta}\right) m_{\lambda}\left(e^{-j \theta}\right) & =0,
\end{aligned}
$$

that is, equation (26) holds.

Case 2 (at a zero): $n_{\alpha}\left(e^{-j \theta}\right)=0$. It follows by coprimeness of $n_{\alpha}(z)$ and $m_{\alpha}(z)$ on the closed unit disk that $m_{\alpha}\left(e^{-j \theta}\right) \neq 0$. Since $e^{-j \theta}$ is not a pole of $p_{\alpha}$, then $p_{\alpha}^{*}\left(e^{-j \theta}\right) p_{\lambda}\left(e^{-j \theta}\right)=-1$. It then follows that $m_{\lambda}\left(e^{-j \theta}\right)=0$, from which it is easy to see that

$$
n_{\alpha}^{*}\left(e^{-j \theta}\right) n_{\lambda}\left(e^{-j \theta}\right)+m_{\alpha}^{*}\left(e^{-j \theta}\right) m_{\lambda}\left(e^{-j \theta}\right)=0,
$$


that is, equation (26) holds.

Case 3 (at a pole): $m_{\alpha}\left(e^{-j \theta}\right)=0$. It follows by coprimeness of $n_{\alpha}(z)$ and $m_{\alpha}(z)$ on the closed unit disk that $n_{\alpha}\left(e^{-j \theta}\right) \neq 0$. We may factorise $m_{\alpha}$ as $m_{\alpha}(z)=$ $\hat{m}_{\alpha}(z)\left[z-e^{-j \theta}\right]^{r_{\alpha}}$ where $\hat{m}_{\alpha}\left(e^{-j \theta}\right) \neq 0$ and $r_{\alpha}$ is a (strictly) positive integer. Equation (27) is equivalent to $T\left(p_{\lambda}\right)=-1$. By equation (25) we see that

$$
\begin{aligned}
T\left(p_{\lambda}\right)= & \frac{2 n_{\alpha}^{*}\left(z^{-1}-e^{-j \theta}\right)^{r_{\alpha}} \hat{m}_{\alpha}^{*} n_{\lambda}}{\left[\left(z^{-1}-e^{-j \theta}\right)^{r_{\alpha}} \hat{m}_{\alpha}^{*} \hat{m}_{\alpha}\left(z-e^{-j \theta}\right)^{r_{\alpha}}+n_{\alpha}^{*} n_{\alpha}\right] m_{\lambda}} \\
& +\frac{\left(z^{-1}-e^{-j \theta}\right)^{r_{\alpha}} \hat{m}_{\alpha}^{*} \hat{m}_{\alpha}\left(z-e^{-j \theta}\right)^{r_{\alpha}}-n_{\alpha}^{*} n_{\alpha}}{\left(z^{-1}-e^{-j \theta}\right)^{r_{\alpha}} \hat{m}_{\alpha}^{*} \hat{m}_{\alpha}\left(z-e^{-j \theta}\right)^{r_{\alpha}}+n_{\alpha}^{*} n_{\alpha}} .
\end{aligned}
$$

In order for $T\left(p_{\lambda}\right)$ to be finite and non-zero, we must have that $m_{\lambda}(z)$ has a zero at $e^{j \theta}$ of order no more than $r_{\alpha}$ (including a possibility of order zero). Recall that $m_{\lambda}$ is real rational and so has a zero of the same order at $e^{-j \theta}$. It then straightforwardly follows that

$$
n_{\alpha}^{*}\left(e^{-j \theta}\right) n_{\lambda}\left(e^{-j \theta}\right)+m_{\alpha}^{*}\left(e^{-j \theta}\right) m_{\lambda}\left(e^{-j \theta}\right)=0,
$$

that is, equation (26) holds.

Note that the inverse transformation for (25) is given by

$$
T^{-1}\left(\bar{p}_{\lambda}\right)=\frac{\left|p_{\alpha}\right|^{2}+1}{2 p_{\alpha}^{*}} \bar{p}_{\lambda}+\frac{\left|p_{\alpha}\right|^{2}-1}{2 p_{\alpha}^{*}} .
$$

Corollary 6.2 (Homotopy "Normalisation"). Provided $p_{\alpha}$ has no poles on the unit circle, the existence of a Vinnicombe homotopy from $\bar{p}_{\alpha}=1$ to $\bar{p}_{\Omega}$ implies the existence of a Vinnicombe homotopy from from $p_{\alpha}$ to $p_{\Omega}$. (Note, however, that at any unit circle zero of $p_{\alpha}$, that is not a zero of $p_{\Omega}$ with the same multiplicity, we have a pole of $\bar{p}_{\Omega}$, so that if there exists such a zero, then $\delta_{\nu}\left(1, \bar{p}_{\Omega}\right)=1$.)

Conversely, provided $p_{\alpha}$ has no zeros on the unit circle, the existence of a Vinnicombe homotopy from $p_{\alpha}$ to $p_{\Omega}$ implies the existence of a Vinnicombe homotopy from from $\bar{p}_{\alpha}=1$ to $\bar{p}_{\Omega}$. (Note, however, that at any unit circle pole of $p_{\alpha}$, that is not a pole of $p_{\Omega}$ with the same multiplicity, we have $1+\bar{p}_{\Omega}=0$, so that if there exists such a pole, then $\left.\delta_{\nu}\left(1, \bar{p}_{\Omega}\right)=1.\right)$

Hence, given a scalar transfer function $p_{\alpha}$, with no poles or zeros on the unit circle, there exists a Vinnicombe homotopy $p_{\lambda}$ from $p_{\alpha}$ to $p_{\Omega}$ if and only if there exists a Vinnicombe homotopy $\bar{p}_{\lambda}$ from $\bar{p}_{\alpha}=1$ to $\bar{p}_{\Omega}=\left[\frac{2 p_{\alpha}^{*}}{1+\left|p_{\alpha}\right|^{2}} p_{\Omega}+\frac{1-\left|p_{\alpha}\right|^{2}}{1+\left|p_{\alpha}\right|^{2}}\right]$.

Proof. Given a Vinnicombe homotopy $p_{\lambda}=\frac{n_{\lambda}}{m_{\lambda}}$ from $p_{\alpha}$ to $p_{\Omega}$, define

$$
\bar{p}_{\lambda}=\left[\frac{2 p_{\alpha}^{*}}{1+\left|p_{\alpha}\right|^{2}} p_{\lambda}+\frac{1-\left|p_{\alpha}\right|^{2}}{1+\left|p_{\alpha}\right|^{2}}\right] .
$$

That $\bar{p}_{\lambda}$ is continuous in $\lambda$ follows from the continuity of $p_{\lambda}$. By Lemma 6.1 , it also follows (provided that $p_{\alpha}$ has no unit circle zeroes, but irrespective of whether 
$p_{\alpha}$ has unit circle poles) that $\bar{n}_{\alpha}^{*}\left(e^{j \theta}\right) \bar{n}_{\lambda}\left(e^{-j \theta}\right)+\bar{m}_{\alpha}^{*}\left(e^{j \theta}\right) \bar{m}_{\lambda}\left(e^{-j \theta}\right) \neq 0$ holds for all $\theta$ and all $\lambda$ because $n_{\alpha}\left(e^{j \theta}\right)^{*} n_{\lambda}\left(e^{-j \theta}\right)+m_{\alpha}\left(e^{j \theta}\right)^{*} m_{\lambda}\left(e^{-j \theta}\right) \neq 0$ holds for all $\theta$ and all $\lambda$. By Lemma 4.5, the only way for the winding number condition to fail is via a failure of the determinant condition.

Hence the existence of a homotopy $p_{\lambda}$ from $p_{\alpha}$ to $p_{\Omega}$ implies the existence of a homotopy $\bar{p}_{\lambda}$ from $\bar{p}_{\alpha}=1$ to $\bar{p}_{\Omega}$. Similar reasoning can be used to prove the reverse implication, but requires that $p_{\alpha}$ possesses no unit circle poles, in order for the valid application of Lemma 6.1.

This implies that for scalar plants without unit circle poles or zeroes, we can consider necessary and sufficient conditions for the existence of homotopies from $\bar{p}_{\alpha}=1$, to $\bar{p}_{\Omega}$ without loss of generality. (Note that there is no suggestion that the transformation $T$ preserves a monotonicity condition on $\delta_{\nu}\left[p_{\lambda}, p_{\alpha}\right]$.)

However for the development of necessary and sufficient conditions, it will be necessary to somewhat generalise the notion of the Cauchy index to allow the critical point in the extended real line over which net crossings are counted to differ from the point at infinity. We present the following definition of a geneneralised Cauchy index and derive some of its properties including the relationship to the standard Cauchy index.

Lemma 6.3. For a given $f(x)=\frac{b(x)}{a(x)}$, a real rational transfer function define the corresponding continuous Brockett angle $\Phi(x)$ by equation (12). Define the generalised Cauchy index $\tilde{\mathcal{I}}_{l}^{u}[\psi, f(\cdot)]$ parametrised by an crossing angle $\psi$ over the real interval $[l, u]$ as the number of net positive strict crossings of $\psi \pm 2 \pi$ of the continuous Brockett angle as $x$ varies over the interval $[l, u]$. Lemma 4.1 states that the (standard) Cauchy index is related to the generalised Cauchy index by $\mathcal{I}_{l}^{u} f(\cdot)=\tilde{\mathcal{I}}_{l}^{u}[\pi, f(\cdot)]$, that is, it is the generalised Cauchy index for $\psi=\pi$.

Furthermore

$$
\begin{aligned}
\tilde{\mathcal{I}}_{l}^{u}[\psi, f(\cdot)] & =\mathcal{I}_{l}^{u} g(\cdot) \\
& =\mathcal{I}_{l}^{u} h(\cdot) \\
& =\tilde{\mathcal{I}}_{l}^{u}[0, k(\cdot)],
\end{aligned}
$$

where

$$
\begin{aligned}
g(x) & =\frac{a(x) \cos \left(\frac{1}{2} \psi\right)+b(x) \sin \left(\frac{1}{2} \psi\right)}{a(x) \sin \left(\frac{1}{2} \psi\right)-b(x) \cos \left(\frac{1}{2} \psi\right)} \\
& =\frac{\cos \left(\frac{1}{2} \psi\right)+f(x) \sin \left(\frac{1}{2} \psi\right)}{\sin \left(\frac{1}{2} \psi\right)-f(x) \cos \left(\frac{1}{2} \psi\right)} \\
h(x) & =\frac{a(x)}{a(x) \tan \left(\frac{1}{2} \psi\right)-b(x)} \\
k(x) & =f(x)-\tan \left(\frac{1}{2} \psi\right),
\end{aligned}
$$


and where the second and third equalities (31) and (32) hold true provided $\cos \left(\frac{1}{2} \psi\right) \neq$ 0 , that is $\psi \neq \pi \pm 2 n \pi$.

Proof. It is easy to see that

$$
\begin{aligned}
g(x) & =\frac{f(x)+\cot \left(\frac{1}{2} \psi\right)}{1-f(x) \cot \left(\frac{1}{2} \psi\right)} \\
2 \tan ^{-1} g(x) & =2 \tan ^{-1} f(x)+(\pi-\psi), \quad \bmod 2 \pi
\end{aligned}
$$

and that $\Phi_{g}(x)$, the Brockett angle associated with $g(x)$ differs from $\Phi_{f}(x)$, the Brockett angle associated with $f(x)$ by an amount $\pi-\psi$. Therefore net positive strict crossings of $\Phi_{g}(x)$ of $\pi$ correspond to net positive strict crossings of $\Phi_{f}(x)$ of $\psi$.

It can also be seen that, provided $\cos \left(\frac{1}{2} \psi\right) \neq 0$, that is $\psi \neq \pi \pm 2 n \pi$

$$
\begin{aligned}
g(x) & =\mathcal{I}_{l}^{u} \frac{a(x)+b(x) \tan \left(\frac{1}{2} \psi\right)}{a(x) \tan \left(\frac{1}{2} \psi\right)-b(x)} \\
& =\frac{a(x) \sec ^{2}\left(\frac{1}{2} \psi\right)-a(x) \tan ^{2}\left(\frac{1}{2} \psi\right)+b(x) \tan \left(\frac{1}{2} \psi\right)}{a(x) \tan \left(\frac{1}{2} \psi\right)-b(x)} \\
& =\frac{a(x) \sec ^{2}\left(\frac{1}{2} \psi\right)}{a(x) \tan \left(\frac{1}{2} \psi\right)-b(x)}-\tan \left(\frac{1}{2} \psi\right) \\
\mathcal{I}_{l}^{u} g(x) & =\mathcal{I}_{l}^{u} \frac{a(x)}{a(x) \tan \left(\frac{1}{2} \psi\right)-b(x)} .
\end{aligned}
$$

Finally, by substituting $\psi=0$ in equation (30) we get

$$
\tilde{\mathcal{I}}_{l}^{u}[0, f]=\mathcal{I}_{l}^{u}\left[-f^{-1}\right]
$$

Hence by identifying $h=\frac{a}{a \tan \left[\frac{1}{2} \psi\right]-b}$ with $-f^{-1}$ in the above

$$
\begin{aligned}
\mathcal{I}_{l}^{u} h & =\tilde{\mathcal{I}}_{l}^{u}\left[0,-h^{-1}\right] \\
& =\tilde{\mathcal{I}}_{l}^{u}[0, k] .
\end{aligned}
$$

Armed with the notion of a generalised Cauchy index, we can now give a necessary and sufficient condition for the existence of a homotopy from 1 to an arbitrary target transfer function.

THEOREM 6.4. There exists a scalar Vinnicombe metric homotopy $p_{\lambda}$ from $p_{\alpha}=1$ to arbitrary $p_{\Omega}$ with $\delta_{\nu}\left(1, p_{\lambda}\right)<1$ if and only if

- $\delta_{\nu}\left(1, p_{\Omega}\right)<1$, and

- $\tilde{\mathcal{I}}_{-1}^{1}\left[-\frac{1}{2} \pi, p_{\Omega}\right]=\tilde{\mathcal{I}}_{-1}^{1}\left[0,1+p_{\Omega}\right]=\mathcal{I}_{-1}^{1} \frac{-1}{1+p_{\Omega}}=0$.

Proof. It is clear from Lemma 6.3, by identifying $f(x)$ and $h(x)$ with respectively $p_{\Omega}$ and $1+p_{\Omega}$, that $\tilde{\mathcal{I}}_{-1}^{1}\left[-\frac{1}{2} \pi, p_{\Omega}\right]=\tilde{\mathcal{I}}_{-1}^{1}\left[0,1+p_{\Omega}\right]=\mathcal{I}_{-1}^{1} \frac{-1}{1+p_{\Omega}}$. That $\tilde{\mathcal{I}}_{-1}^{1}\left[-\frac{1}{2} \pi, p_{\Omega}\right]=0$ is equivalent to, in the $x$-interval $[-1,1]$, the number of net crossings of the continuous Brockett angle $\Phi_{\Omega}(x)$ corresponding to $p_{\Omega}$ of the value $-\frac{1}{2} \pi \pm 2 n \pi$ being equal to 
zero. Note also that $\Phi_{\alpha}$, the continuous Brockett angle corresponding to $p_{\alpha}=1$ is $2 \tan ^{-1} 1=\frac{1}{2} \pi$.

We now show that the conditions in the Lemma hypothesis are necessary for the existence of a homotopy. Clearly the first condition is necessary since the subunitary condition $\delta_{\nu}\left(1, p_{\lambda}\right)<1$ must hold, even at the final end point $\lambda=\Omega$.

For the second condition, note that since $\Phi_{\alpha}(x)=\frac{1}{2} \pi$ for all $x$, then by Lemma 4.6, if a homotopy $p_{\lambda}$ exists from $p_{\alpha}=1$ to $p_{\Omega}$, then both the Brockett angles $\Phi_{\Omega}(1)$ and $\Phi_{\Omega}(-1)$ must lie in the interval $\left(\frac{1}{2} \pi-\pi, \frac{1}{2} \pi+\pi\right)=\left(-\frac{1}{2} \pi, \frac{3}{2} \pi\right)$. This is sufficient to ensure that $\tilde{\mathcal{I}}_{-1}^{1}\left[-\frac{1}{2} \pi, p_{\Omega}\right]=0$.

We now show that provided the lemma hypotheses hold, there exists a homotopy from $p_{\alpha}=1$ to $p_{\Omega}$ via $p_{\lambda}$ such that $\delta_{\nu}\left(1, p_{\lambda}\right)<1$ for all $\lambda$. Since $\delta_{\nu}\left(1, p_{\Omega}\right)<1$ it follows that

$$
\begin{aligned}
\operatorname{wno}\left(1+p_{\Omega}\right)+\breve{\eta}\left(p_{\Omega}\right) & =0, \\
\breve{\mathcal{Z}}\left(1+p_{\Omega}\right)-\breve{\eta}\left(1+p_{\Omega}\right)+\breve{\eta}\left(p_{\Omega}\right) & =0, \\
\breve{\mathcal{Z}}\left(1+p_{\Omega}\right) & =0 .
\end{aligned}
$$

(Recall that $\breve{\mathcal{Z}}(X)$ denotes the number of zeros of transfer function $X$, including multiplicity in the open interior of the unit disk.) Let

$$
1+p_{\Omega}(z)=\frac{\tilde{n}_{\Omega}(z)}{\tilde{m}_{\Omega}(z)},
$$

such that $\tilde{n}_{\Omega}$ and $\tilde{m}_{\Omega}$ are polynomials and $\tilde{n}_{\Omega}(z)=K \prod_{k}\left(1-\frac{1}{\zeta_{k}} z\right)$ has no zeros in the open unit disk and $\tilde{n}_{\Omega}(0)=K>0$. We then define for $\lambda \in[0,1]$

$$
\begin{aligned}
\tilde{n}_{\lambda}(z) & =[2+(K-2) \lambda] \prod_{k}\left(1-\frac{\lambda}{\zeta_{k}} z\right), \\
\tilde{m}_{\lambda}(z) & =(1-\lambda)+\lambda \tilde{m}_{\Omega}(z), \\
n_{\lambda}(z) & =\tilde{n}_{\lambda}(z)-\tilde{m}_{\lambda}(z), \\
m_{\lambda}(z) & =\tilde{m}_{\lambda}(z) .
\end{aligned}
$$

Then we define a homotopy $p_{\lambda}$ as

$$
p_{\lambda}=\frac{n_{\lambda}}{m_{\lambda}}=\frac{\tilde{n}_{\lambda}}{\tilde{m}_{\lambda}}-1 .
$$

If follows by construction that $\tilde{n}_{\lambda}(z)$ possesses no zeroes in the open unit disk and therefore for $\lambda \in[0,1]$ we have $\tilde{\mathcal{I}}_{-1}^{1}\left[0, \frac{\tilde{n}_{\lambda}}{\tilde{m}_{\lambda}}\right]=\tilde{\mathcal{I}}_{-1}^{1}\left[0,1+p_{\lambda}\right]=0$, because we have no crossing of $\psi=0$ of the continuous Brockett angle corresponding to $\frac{\tilde{n}_{\lambda}}{\tilde{m}_{\lambda}}$ in the real interval $z \in[-1,1]$. It also follows that $\tilde{n}_{\lambda}$ and $\tilde{m}_{\lambda}$ possess no common zeroes in the open unit disk, and that the same holds for $n_{\lambda}$ and $m_{\lambda}$. We then have that

$$
\begin{aligned}
\operatorname{wno}\left(1+p_{\lambda}\right)+\breve{\eta}\left(p_{\lambda}\right) & =\operatorname{wno}\left(\frac{\tilde{n}_{\lambda}}{\tilde{m}_{\lambda}}\right)+\breve{\eta}\left(\frac{\tilde{n}_{\lambda}}{\tilde{m}_{\lambda}}\right) \\
& =\mathcal{Z}\left(\frac{\tilde{n}_{\lambda}}{\tilde{m}_{\lambda}}\right)=0 .
\end{aligned}
$$


Finally, for all $\lambda$, we have

$$
\begin{aligned}
\underset{|z|=1}{\operatorname{ess} \sup } \frac{\left|p_{\lambda}(z)-1\right|}{\sqrt{2\left(1+\left|p_{\lambda}(z)\right|^{2}\right)}} & =\underset{|z|=1}{\operatorname{essup}} \frac{\left|\frac{n_{\lambda}}{m_{\lambda}}-1\right|}{\sqrt{2\left(\left|m_{\lambda}(z)\right|^{2}+\left|n_{\lambda}(z)\right|^{2}\right.}} \\
& =\underset{|z|=1}{\operatorname{essup} \sup } \frac{\left|n_{\lambda}(z)-m_{\lambda}(z)\right|^{2}}{2\left(\left|m_{\lambda}(z)\right|^{2}+\left|n_{\lambda}(z)\right|^{2}\right)} .
\end{aligned}
$$

Now observe that

$$
\begin{aligned}
\left|\frac{\tilde{n}_{\lambda}(z)}{\tilde{m}(z)}\right|^{2} & =\frac{\left|n_{\lambda}(z)+m_{\lambda}(z)\right|^{2}}{\left|m_{\lambda}\right|^{2}} \\
& >0, \quad \text { for all }|z|=1 .
\end{aligned}
$$

So for all $|z|=1$ the following holds.

$$
\begin{aligned}
\left|n_{\lambda}(z)+m_{\lambda}(z)\right|^{2} & >0 \\
\left|n_{\lambda}(z)\right|^{2}+\left|m_{\lambda}(z)\right|^{2} & >-n_{\lambda}(z)^{*} m_{\lambda}(z)-n_{\lambda}(z) m_{\lambda}(z)^{*}, \\
2\left|n_{\lambda}(z)\right|^{2}+2\left|m_{\lambda}(z)\right|^{2} & >\left|n_{\lambda}(z)\right|^{2}+\left|m_{\lambda}(z)\right|^{2}-n_{\lambda}(z)^{*} m_{\lambda}(z)-n_{\lambda}(z) m_{\lambda}(z)^{*} .
\end{aligned}
$$

Hence,

$$
1>\underset{|z|=1}{\operatorname{essup}} \frac{\left|n_{\lambda}(z)-m_{\lambda}(z)\right|^{2}}{2\left(\left|m_{\lambda}(z)\right|^{2}+\left|n_{\lambda}(z)\right|^{2}\right)} .
$$

Since the winding number condition (33) holds it follows that $\delta_{\nu}\left(1, p_{\lambda}\right)<1$.

Now that we have necessary and sufficient conditions for the existence of a homotopy from the fixed initial transfer function $p_{\alpha}=1$ to an arbitrary final transfer function, we can use the normalisation transformation of Lemma 6.1 to given necessary and sufficient conditions for the existence of a homotopy from a transfer function $p_{\alpha}$ that possesses no unit circle poles but is otherwise arbitrary.

REMARK 6.5. By Lemma 6.3 the condition $\tilde{\mathcal{I}}_{-1}^{1}\left[-\frac{1}{2} \pi, p_{\Omega}\right]=\tilde{\mathcal{I}}_{-1}^{1}\left[0,1+p_{\Omega}\right]=$ $\mathcal{I}_{-1}^{1} \frac{1}{1+p_{\Omega}}=0$ in the hypothesis of Theorem 6.4 is equivalent to $\mathcal{I}_{-1}^{1} \frac{1-p_{\Omega}}{1+p_{\Omega}}=\tilde{\mathcal{I}}_{-1}^{1}[\pi$, $\left.\frac{1}{1+p_{\Omega}}\right]=0$.

Corollary 6.6 (Main Result). Provided that $p_{\alpha}$ has no poles or zeros on the unit circle, there exists a scalar Vinnicombe metric homotopy $p_{\lambda}$ from $p_{\alpha}$ to $p_{\Omega}$ with $\delta_{\nu}\left(p_{\alpha}, p_{\lambda}\right)<1$ if and only if

- $\delta_{\nu}\left(p_{\alpha}, p_{\Omega}\right)<1$, and

- $\mathcal{I}_{-1}^{1}\left[\frac{1+p_{\alpha}^{*} p_{\alpha}}{1+p_{\alpha}^{*} p_{\Omega}}\right]=0$.

Proof. This is a consequence of Corollary 6.2 and Theorem 6.4, using the identity $\tilde{\mathcal{I}}_{-1}^{1}\left[0,1+p_{\Omega}\right]=\mathcal{I}_{-1}^{1} \frac{1}{1+p_{\Omega}}$.

We first apply Corollary 6.2. Since $p_{\alpha}$ has no poles on the unit circle, the existence of a scalar Vinnicombe metric homotopy $p_{\lambda}$ from $p_{\alpha}$ to $p_{\Omega}$ with $\delta_{\nu}\left(p_{\alpha}, p_{\lambda}\right)<1$ is equivalent to the existence of a Vinnicombe metric homotopy $\bar{p}_{\lambda}$ from 1 to

$$
\bar{p}_{\Omega}=\left[\frac{2 p_{\alpha}^{*}}{1+\left|p_{\alpha}\right|^{2}} p_{\Omega}+\frac{1-\left|p_{\alpha}\right|^{2}}{1+\left|p_{\alpha}\right|^{2}}\right]
$$


By Theorem 6.4 this will hold if and only if

- $\delta_{\nu}\left(1, \bar{p}_{\Omega}\right)<1$, and

- $\tilde{\mathcal{I}}_{-1}^{1}\left[-\frac{1}{2} \pi, \bar{p}_{\Omega}\right]=\tilde{\mathcal{I}}_{-1}^{1}\left[0,1+\bar{p}_{\Omega}\right]=\mathcal{I}_{-1}^{1} \frac{-1}{1+\bar{p}_{\Omega}}=0$.

Note also that $\delta_{\nu}\left(1, \bar{p}_{\Omega}\right)<1$ is equivalent to $\delta_{\nu}\left(p_{\alpha}, p_{\Omega}\right)<1$ and that

$$
\begin{aligned}
1+\bar{p}_{\Omega} & =1+\frac{2 p_{\alpha}^{*} p_{\Omega}+1-\left|p_{\alpha}\right|^{2}}{1+\left|p_{\alpha}\right|^{2}} \\
& =2 \frac{1+p_{\alpha}^{*} p_{\Omega}}{1+p_{\alpha}^{*} p_{\alpha}}
\end{aligned}
$$

so that $\mathcal{I}_{-1}^{1} \frac{-1}{1+\bar{p}_{\Omega}}=0$ is equivalent to $\mathcal{I}_{-1}^{1}-\frac{1}{2} \frac{1+p_{\alpha}^{*} p_{\alpha}}{1+p_{\alpha}^{*} p_{\Omega}}=0$ and $\mathcal{I}_{-1}^{1}\left[\frac{1+p_{\alpha}^{*} p_{\alpha}}{1+p_{\alpha}^{*} p_{\Omega}}\right]=0$.

7. Conclusion. We have given necessary and sufficient conditions for the existence of a Vinnicombe metric homotopy between two given scalar transfer functions, provided at least one of the end points has no marginally unstable poles. The necessary and sufficient conditions are equivalent to the satisfaction of a winding number condition and a Cauchy index condition. The homotopy is subunitary, in the sense that $\delta_{\nu}\left(p_{\alpha}, p_{\lambda}\right)$ is less than unity at all values of the homotopy parameter $\lambda$, although there is no suggestion that the homotopy is necessarily monotonic. Lemma 4.6 gives an equivalent condition, in terms of a difference in the continuous Brockett angle over the interval $[-1,1]$, which is shown to be necessary for the existence of a subunitary homotopy, even For the case where the initial transfer function does possess marginally unstable poles. The authors conjecture that this condition is also sufficient for the existence of a homotopy, but have been unable to prove this. It is possible that a perturbation argument might be used to demonstrate this sufficiency.

This paper therefore, virtually settles the question of the relationship between the winding number condition and the existence of a subunitary Vinnicombe metric homotopy for scalar transfer functions, except for the special case of marginally unstable transfer functions. On the basis of the results presented here, taken together with those in [4] which gives a constructive subunitary (and arbitrarily close to monotonic) homotopy between two given multivariable transfer functions which satisfy a winding number condition, it is fair to say that this relationship is now reasonably well understood.

\section{REFERENCES}

[1] B. D. O. Anderson And T. S. Brinsmead, Not all Vinnicombe metric neighbourhoods are homotopically connected, Proceedings on Information, Decision and Control, Adelaide, February, 2002.

[2] B. D. O. Anderson, T. S. Brinsmead, and F. De Bruyne, The Vinnicombe metric for nonlinear operators, IEEE Transactions on Automatic Control, 47:9(2002), pp. 1450-1465.

[3] B. D. O. Anderson and F. De Bruyne, On a nonlinear generalisation of the $\nu$-gap metric, Proc. $38^{\text {th }}$ IEEE Conf. on Decision and Control, pp. 3851-3856, 1999. 
[4] T. S. Brinsmead and B. D. O. Anderson, Homotopy for the $\nu$-gap, Journal of Communications in Information and Systems, 1:4(2001), pp. 333-380.

[5] R. W. Brockett. Some geometric questions in the theory of linear systems, IEEE Transactions on Automatic Control, AC-21:4(1976), pp. 449-455.

[6] M. Cantoni and G. Vinnicombe, Linear Feedback Systems and the Graph Toplogy, IEEE Transactions on Automatic Control, AC-47:5(2002), pp. 710-719.

[7] F. De Mari and R. Ober Topological Aspects of Robust Control, In D. Hinrichsen and B. Martensson Control of Uncertain Systems, Birkhäuser, Boston, 1990, pp. 57-57 .

[8] A. K. El-Sakkary, The gap metric: Robustness of stabilization of feedback systems, IEEE Trans. Auto. Control, AC-30:3(1985), pp. 240-247.

[9] F. Gantmacher, The Theory of Matrices, Chelsea Publishing Company, New York, 1959.

[10] T. T. Georgiou and M. C. Smith, Optimal robustness in the gap metric, IEEE Trans. Auto. Control, 35:6(1990), pp. 673-687.

[11] T. T. Georgiou And M. C. Smith, Robustness analysis of nonlinear feedback systems: An input-output approach, IEEE Trans. Auto. Control, 42:9(1997), pp. 1200-1219.

[12] M. R. James, M. C. Smith, and G. Vinnicombe, Gap metrics, representations, and nonlinear robust stability, Proc. $39^{\text {th }}$ IEEE Conf. on Decision and Control, pp. 2557-2562, 2000.

[13] M. Vidyasagar, Control System Synthesis: A Factorization Approach. MIT Press, Cambridge, Massachusetts, 1985.

[14] G. Vinnicombe, Frequency domain uncertainty and the graph topology, IEEE Trans. Auto. Control, 38:9(1993), pp. 1371-1383.

[15] G. Vinnicombe, A $\nu$-gap distance for uncertain and nonlinear systems, Proc. $38^{\text {th }}$ IEEE Conf. on Decision and Control, pp. 2557-2562, 1999.

[16] G. Vinnicombe, On closed-loop objectives and guaranteed robustness properties, Proc. $35^{\text {th }}$ IEEE Conf. on Decision and Control, pp. 1869-1874, 1996.

[17] G. Vinnicombe, Uncertainty and Feedback: $\mathcal{H}_{\infty}$ Uncertainty and the $\nu$-gap metric. Imperial College Press, London, 2001. 
THOMAS S. BRINSMEAD AND BRIAN D. O. ANDERSON 